\title{
Neutrino Emissions in All Flavors up to the Pre-bounce of Massive Stars and the Possibility of Their Detections
}

\author{
Chinami Kato ${ }^{1}$ (10), Hiroki Nagakura ${ }^{2}$ (10), Shun Furusawa ${ }^{3,4}$, Koh Takahashi $^{5,6}$, Hideyuki Umeda ${ }^{5}$, Takashi Yoshida ${ }^{5}$, \\ Koji Ishidoshiro ${ }^{7}$ (1), and Shoichi Yamada ${ }^{1,8}$ (1) \\ ${ }^{1}$ School of Advanced Science and Engineering, Waseda University, 3-4-1, Okubo, Shinjuku, Tokyo 169-8555, Japan; chinami@heap.phys.waseda.ac.jp \\ ${ }^{2}$ TAPIR, Walter Burke Institute for Theoretical Physics, Mailcode 350-17, California Institute of Technology, Pasadena, CA 91125, USA \\ ${ }^{3}$ Frankfurt Institute for Advanced Studies, J.W. Goethe University, D-60438 Frankfurt am Main, Germany \\ ${ }^{4}$ Interdisciplinary Theoretical Science Research Group, RIKEN, Wako, Saitama 351-0198, Japan \\ ${ }^{5}$ Department of Astronomy, The University of Tokyo, Tokyo 113-0033, Japan \\ ${ }_{7}^{6}$ Argelander Institute for Astronomy, Auf dem Hügel 71, D-53121 Bonn, Germany \\ ${ }^{7}$ Research Center for Neutrino Science, Tohoku University, Sendai 980-8578, Japan \\ ${ }^{8}$ Advanced Research Institute for Science and Engineering, Waseda University, 3-4-1, Okubo, Shinjuku, Tokyo 169-8555, Japan \\ Received 2017 April 18; revised 2017 September 6; accepted 2017 September 7; published 2017 October 10
}

\begin{abstract}
This paper is a sequel to our 2015 paper, Kato et al., which calculated the luminosities and spectra of electron-type anti-neutrinos $\left(\bar{\nu}_{e}\right)$ from the progenitors of core-collapse supernovae. Expecting that the capability to detect electron-type neutrinos $\left(\nu_{e}\right)$ will increase dramatically with the emergence of liquid-argon detectors such as DUNE, we broaden the scope in this study to include all flavors of neutrinos emitted from the pre-bounce phase. We pick up three progenitor models of electron capture supernovae (ECSNe) and iron-core collapse supernovae (FeCCSNe). We find that the number luminosities reach $\sim 10^{57} \mathrm{~s}^{-1}$ and $\sim 10^{53} \mathrm{~s}^{-1}$ at maximum for $\nu_{e}$ and $\bar{\nu}_{e}$, respectively. We also estimate the numbers of detection events at terrestrial neutrino detectors including DUNE, taking flavor oscillations into account and assuming the distance to the progenitors to be $200 \mathrm{pc}$. It is demonstrated that $\bar{\nu}_{e}$ from the ECSN progenitor will be undetected at almost all detectors, whereas we will be able to observe $\gtrsim 15,900 \nu_{e}$ at DUNE for the inverted mass hierarchy. From the FeCCSN progenitors, the number of $\bar{\nu}_{e}$ events will be largest for JUNO, 200-900 $\bar{\nu}_{e}$, depending on the mass hierarchy, whereas the number of $\nu_{e}$ events at DUNE is $\gtrsim 2100$ for the inverted mass hierarchy. These results imply that the detection of $\bar{\nu}_{e}$ is useful to distinguish progenitors of FeCCSNe from those of ECSNe, while $\nu_{e}$ will provide us with detailed information on the collapse phase regardless of the type and mass of the progenitor.
\end{abstract}

Key words: neutrinos - stars: evolution - stars: massive - supernovae: general

\section{Introduction}

Massive stars with $M_{\text {ZAMS }} \gtrsim 8 M_{\odot}$ are supposed to be the progenitors of core-collapse supernovae (CCSNe), which are violent explosions at the end of their lives. The explosion is instigated by the collapse of a central core, which is followed by the formation of a shock wave at core bounce. If the shock wave runs through the central core and propagates through the outer envelopes up to the stellar surface, these envelopes are ejected and a compact remnant is left behind at the center. The mechanism for getting the shock wave out of the core has been explored for a long time but has not yet been settled (Janka 2012; Kotake et al. 2012, and references therein). One of the current focus is some features in the structures of progenitors such as the compactness of the core and convective activities in the envelopes (Couch et al. 2015; Müller 2015).

We consider two types of progenitors that are supposed to produce CCSNe: in a majority of cases, these progenitors produce a core mainly composed of iron (Fe core), which collapses when a certain density or temperature is reached; in the other case, which comprises $~ 5 \%$ of all CCSNe according to a recent study (Doherty et al. 2017), gravitational contraction already starts after a core consisting of oxygen and neon (ONe core) is formed via carbon burning (C burning) and grows to a critical mass, $M_{\text {core }}=1.376 M_{\odot}$ (Woosley et al. 2002). The initial stellar mass on the main sequence is the main factor that determines which is obtained in the end: stars on the lightest end of massive stars $\left(\sim 8-10 M_{\odot}\right)$ will lead to the latter and more massive stars will produce the former (Nomoto \& Hashimoto 1988; Umeda et al. 2012; Jones et al. 2013).

In fact, if a star is massive enough $\left(M_{\text {ZAMS }} \gtrsim 10 M_{\odot}\right)$, then the temperature at the center reaches the ignition point of $\mathrm{Ne}$ or $\mathrm{O}$, synthesizing iron-group elements through $\mathrm{Si}$ burnings. Electron captures (ECs) on and/or photodissociations of these heavy nuclei trigger the gravitational collapse of the $\mathrm{Fe}$ core. This evolution toward a collapse and the ensuing explosion are referred to as "iron-core collapse supernovae (FeCCSNe)." For the lighter masses, on the other hand, electrons are more degenerate in the $\mathrm{ONe}$ core, and their pressure can support the core even at vanishing temperatures. The mass of the $\mathrm{ONe}$ core increases through shell $\mathrm{C}$ burnings, however, and if it exceeds the critical value $M_{\text {core }}=1.376 M_{\odot}$, at which the central density reaches the threshold for $\mathrm{EC}$ on ${ }^{24} \mathrm{Mg}\left(\log _{10} \rho_{c} /\left[\mathrm{g} \mathrm{cm}^{-3}\right]=9.88\right)$, then the core begins to contract, losing the pressure support from electrons (Takahashi et al. 2013). This leads in turn to EC on ${ }^{20} \mathrm{Ne}$, accelerating the contraction and eventually igniting $\mathrm{O}$ and $\mathrm{Ne}$. The $\mathrm{O}$ and $\mathrm{Ne}$ burnings propagate as a deflagration wave, establishing nuclear statistical equilibrium (NSE) in its wake. Neutrinos are then emitted copiously via EC reactions on iron-group elements and free protons, which eventually trigger the collapse of the $\mathrm{ONe}$ core that proceeds on a dynamical timescale. In this paper, we call this mode of collapse and the subsequent CCSNe either an "electron capture supernova (ECSNe)" or "ONe-core collapse supernovae (ONeCCSNe)." The resultant supernova explosions with an explosion energy of $\sim 10^{50}$ erg are supposed to be weaker than $\mathrm{FeCCSNe}$ with $\sim 10^{51}$ erg (Kitaura et al. 2006; 
Janka et al. 2007). In fact, SN 1054, which produced the Crab pulsar, may be one such ECSN (Nomoto et al. 1982; Tominaga et al. 2013).

Neutrinos play an important role in both progenitor evolutions and the supernova explosion itself. In fact, the neutrino heating mechanism is currently thought to be the most promising scenario to revive a stalled shock and produce a successful explosion. CCSNe are also one of the most important cosmic neutrino sources from an observational point of view (Raffelt 2012) as corroborated by the observation of neutrinos from SN 1987A at terrestrial neutrino detectors such as Kamiokande (Hirata et al. 1987; Arnett et al. 1989). These neutrinos are mostly emitted in the cooling phase of protoneutron stars (PNSs), which follows the shock revival and lasts for $\sim 10$ s (Sato \& Suzuki 1987; Burrows \& Lattimer 1988; Fischer et al. 2012). Before core collapse, on the other hand, neutrinos dominate photons in the stellar cooling after $\mathrm{C}$ burning. These neutrinos are called "pre-supernova (pre-SN) neutrinos." As the central temperature and density increase in the progenitor, the number and energy of pre-SN neutrinos also increase (Odrzywolek \& Heger 2010).

Neutrinos are emitted via thermal pair processes and nuclear weak interactions. Among the former, electron-positron pair annihilations and plasmon decays are important in the late phase of stellar evolution (Itoh et al. 1996; Kato et al. 2015). Odrzywolek et al. (2004) were the first to pay attention to neutrino emissions via electron-positron pair annihilation and to point out that they may be observable during the Si-burning phase if the progenitor is located at a distance $\lesssim 1 \mathrm{kpc}$. Later, they also investigated the energy spectrum of plasmon decay (Odrzywolek 2007). Odrzywolek (2009) and Patton et al. (2017) pointed out that neutrino emissions via nuclear weak processes, such as $\beta^{-}$decay, may become dominant just prior to collapse. Misch \& Fuller (2016) discussed the importance of excited states in both parent and daughter nuclei in these processes.

Kato et al. (2015, hereafter "Paper I") took into account realistic stellar evolutions that lead to both FeCCSNe and ONeCCSNe. They showed that these two types of supernova progenitors can be distinguished by the detection (or nondetection) of their pre-SN neutrinos. Yoshida et al. (2016) investigated in more detail the pre-SN neutrino luminosities and the cumulative numbers of detection events as a function of time for FeCCSN progenitors. They demonstrated that pre-SN neutrinos can be a useful probe into Si burning, which occurs deep inside massive stars, if they are observed by the nextgeneration detectors such as JUNO and Hyper-Kamiokande.

In the observation of $\bar{\nu}_{e}$, the detectors in operation at present, both water Cherenkov and liquid scintillation types, mainly employ the inverse $\beta$ decay, whose cross-section dominates those of other reactions such as elastic scattering on electrons. It is $\nu_{e}$, however, that are produced in the largest quantity as a result of EC. It is therefore nice, from an observational point of view, that new detectors that have capabilities of detecting $\nu_{e}$ may become available in the near future. The Deep Underground Neutrino Observatory, or DUNE, is a liquid-argon detector currently planned to be constructed in 10 years at SURF (Sanford Underground Research Facility; DUNE collaboration 2016). It will deploy four detectors, each filled with $10 \mathrm{kt}$ of liquid argon. Although the detection of supernova neutrinos emitted after core bounce is one of the main targets of DUNE, it should be noted that its energy threshold will be low enough $(\sim 5 \mathrm{MeV})$ to detect $\nu_{e}$ in the pre-SN phase. In this paper, we calculate the $\nu_{e}$ produced via both thermal and weak processes and discuss their detectability. Although in principle the Helium and Lead Observatory (HALO) experiment at SNOLAB can also detect $\nu_{e}$ with helium and lead, it is not suitable for the detection of pre-SN neutrinos because of its small volume and high energy threshold (Zuber 2015).

The neutrino emissions at different phases, i.e., the progenitor phase, pre-/post-bounce phases, and PNS-cooling phase, have been investigated separately so far. Considering, however, the recent progress in the numerical modeling of CCSNe, in which we rather commonly observe successful explosions, we believe that these phases should be handled consistently, based on successful supernova models. This paper is the first step in this direction, and we attempt to calculate consistently and seamlessly the neutrino emissions from the progenitor stage up to the pre-bounce time, at which point the central density becomes $\log _{10} \rho_{c} /\left[\mathrm{g} \mathrm{cm}^{-3}\right]=13$. The subsequent evolutions of the same models will be studied later.

The organization of the paper is as follows: the progenitor models for ECSNe and FeCCSNe are briefly described in Section 2, the calculations of the luminosities and spectra of neutrinos are summarized for individual processes in Section 3, the results are presented in Section 4, and finally the summary and discussions are given in Section 5.

\section{Models}

In this paper, we consider neutrino emissions during both the quasi-static evolutions of the progenitors and the hydrodynamical core collapse. We stop the calculations when the central density reaches $\log _{10} \rho_{c} /\left[\mathrm{g} \mathrm{cm}^{-3}\right]=13$. For the former, we use the stellar evolution models as described in Section 2.1, whereas for the latter, we conduct one-dimensional simulations under spherical symmetry, solving radiation-hydrodynamics equations as explained in Section 2.2. Note that we need to take properly into account neutrino transport in the core once the density becomes high enough to trap neutrinos. The two evolutionary phases are connected when the central density $\log _{10} \rho_{c} /\left[\mathrm{g} \mathrm{cm}^{-3}\right]=10.3$ for FeCCSNe and 10.1 for ECSNe, respectively.

\subsection{Quasi-static Evolutions of Progenitors}

We employ three progenitor models with $M_{\text {ZAMS }}=9,12$, and $15 M_{\odot}$, which were calculated anew by Takahashi et al. (2013, 2016). The first one produces an ONe core that is supposed to explode as an ECSN, while the last two models explode as FeCCSNe if they were successful. We employ the $9 M_{\odot}$ model instead of the $8.4 M_{\odot}$ model adopted in Paper I, since K. Takahashi et al. (2017, in preparation) have improved the treatment of convective overshooting in the $9 M_{\odot}$ model and are currently investigating in detail the core collapse and subsequent explosion of the same model. The latter two models with 12 and $15 M_{\odot}$ are indeed identical to those employed in Yoshida et al. (2016) but calculated until the central temperature reaches $10^{10} \mathrm{~K}$ with hydrodynamics taken into account.

Here we briefly summarize the evolutions of these models. Figure 1 shows the evolutions of the central density and temperature of the progenitors. The solid lines represent the results of the quasi-static stellar-evolutionary calculations or the "progenitor phase," whereas the dashed lines correspond to those of the core-collapse simulations or the "collapse phase." 


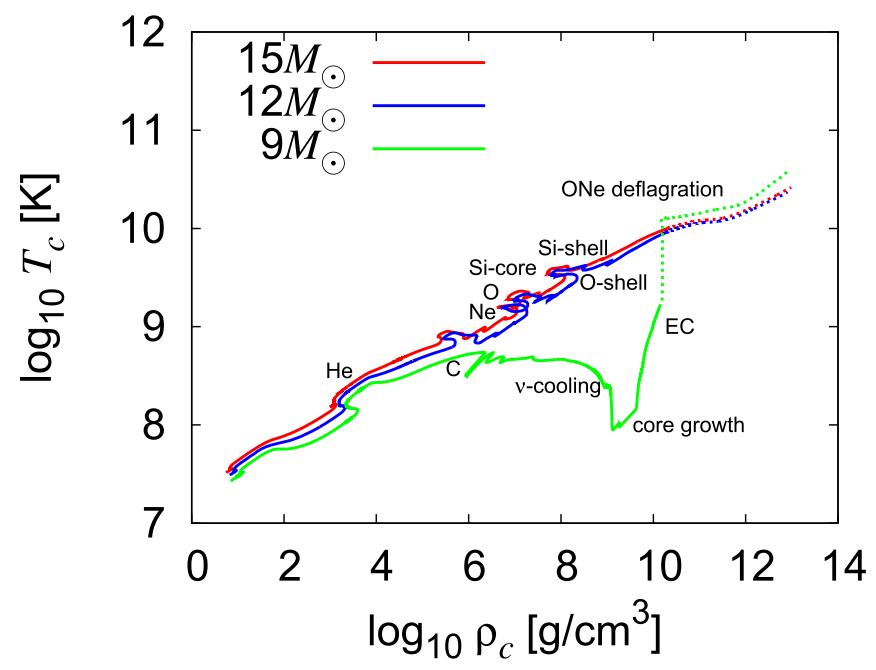

Figure 1. Evolutionary paths of the central density and temperature for three progenitors. The red, blue, and green curves correspond to the 15, 12, and $9 M_{\odot}$ models, respectively. The evolutions in both the progenitor phase (solid lines) and collapse phase (dotted lines) are presented. The initiation points of some major nuclear burnings as well as the evolutionary stages defined by Takahashi et al. (2013) for the ONe-core progenitors are marked with labels.

In this figure, we also mark the initiation points of major nuclear-burning stages, which are defined to be the points when the relevant element is ignited at the center; for the case of the $\mathrm{ONe}$ core, more detailed evolutionary stages are indicated as well, which are defined in Takahashi et al. (2013). We see that the two types of progenitors are not much different up to the end of $C$ burning $\left(\log _{10} \rho /\left[\mathrm{g} \mathrm{cm}^{-3}\right] \sim 6\right)$. After that, however, the evolutionary paths deviate remarkably from each other. The progenitors with 12 and $15 M_{\odot}$ proceed further to burn heavier nuclei stably under the supports not only of thermal but also of degenerate pressures, and their central densities and temperatures increase gradually up to the collapse. In the case of the progenitor with $M_{\mathrm{ZAMS}}=9 M_{\odot}$, on the other hand, $\mathrm{Ne}$ burning does not occur immediately, since the temperature does not become high enough after the $\mathrm{C}$ burning. The core is cooled by neutrino emissions, and the central temperature is lowered as the $\mathrm{ONe}$ core grows via shell $\mathrm{C}$ burning, and the central density increases. When it reaches the critical value $\left(\log _{10} \rho /\left[\mathrm{g} \mathrm{cm}^{-3}\right]=9.88\right)$ for $\mathrm{EC}$ on ${ }^{24} \mathrm{Mg}$, then the core starts to contract with a shorter timescale and the central temperature also begins to rise again. The contraction is considerably accelerated when EC on ${ }^{20} \mathrm{Ne}$ sets in at $\log _{10} \rho /\left[\mathrm{g} \mathrm{cm}^{-3}\right]=10.3$, accompanied by a rapid rise of the central temperature. Finally, $\mathrm{Ne}$ and $\mathrm{O}$ are ignited at the center almost simultaneously, and the flame front starts to propagate outward as a deflagration. The temperature increases drastically and NSE is established soon after the passage of the burning front.

The evolution of the central density for the three progenitors is shown in Figure 2. The origin of the time coordinate corresponds to the time when the hydrodynamical calculations are initiated. When a new nuclear burning starts, the core expands and the central density is lowered a bit. It is also evident in this figure that the pre-collapse evolution of the $9 M_{\odot}$ progenitor is qualitatively different from the other two.

In Figure 3, the radial profiles of the density $\rho$, temperature $T$, electron degeneracy $\mu_{e} / T$, where $\mu_{e}$ is the chemical potential of the electron, and electron fraction $Y_{e}$ are plotted. The horizontal axis is the mass coordinate in solar mass units.

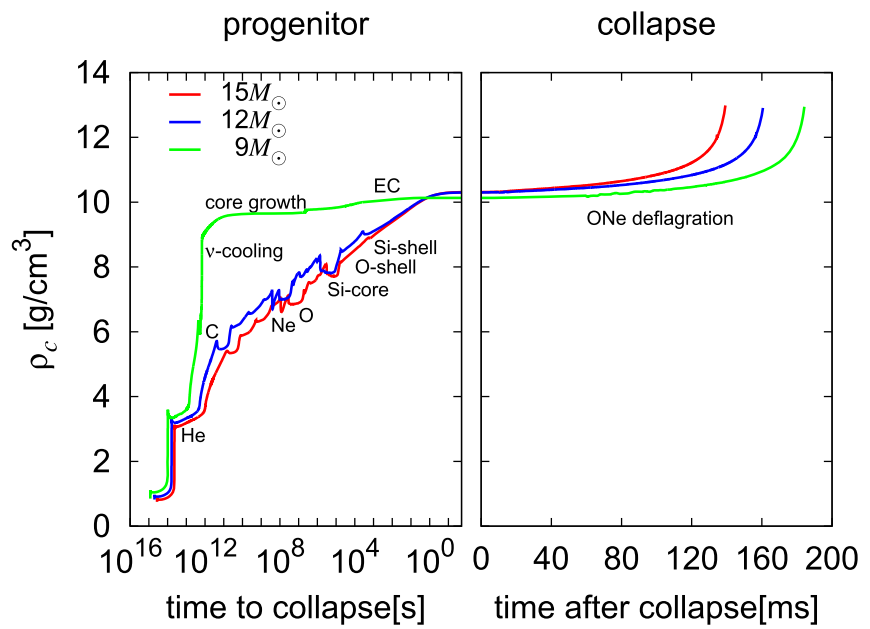

Figure 2. Evolutions of the central density as functions of time for the three progenitors. The red, blue, and green curves correspond to the 15, 12, and $9 M_{\odot}$ models, respectively. The origin of the horizontal axis corresponds to the time when the dynamical simulations started. The initiation points of some major nuclear burnings as well as the evolutionary stages for the ONe-core progenitors are marked with labels.

Different colors correspond to the different times when the central densities are $\log _{10} \rho_{c} /\left[\mathrm{g} \mathrm{cm}^{-3}\right]=6,8,10,12$, and 13 . It is clear from the comparison between the progenitors of $\mathrm{FeCCSNe}$ and those of ECSNe that the temperature profiles become qualitatively different at $\log _{10} \rho_{c} /\left[\mathrm{g} \mathrm{cm}^{-3}\right]=8$. In the case of the $9 M_{\odot}$ progenitor, the central part of the core is cooler than the outer part because of neutrino cooling via plasmon decay. The degeneracy parameter $\mu_{e} / T$ is accordingly higher than that in the 12 and $15 M_{\odot}$ models. Rather high electron fractions $\left(Y_{e} \sim 0.498\right)$ at early times are a noteworthy feature for the ECSN progenitor. Although EC reactions trigger core contraction, the change in $Y_{e}$ is rather minor $\left(\Delta Y_{e} \sim 0.008\right)$ in this phase, and the main reduction in $Y_{e}$ occurs only after NSE is established by $\mathrm{O}+\mathrm{Ne}$ deflagration.

\subsection{Core Collapse}

Once accelerated gravitational contraction happens after EC on ${ }^{20} \mathrm{Ne}$ in the core, we have to abandon the quasi-static approximation and need to solve the hydrodynamical equations numerically. As explained earlier, interactions of neutrinos with matter become non-negligible as the density increases and neutrinos are eventually trapped in the core. Then, we need to take into account the transport of neutrinos appropriately. We thus employ the one-dimensional hydrodynamical code with a Boltzmann solver developed by Nagakura et al. $(2014,2016)$ to follow the evolution of the core collapse. The hydrodynamics solver is explicit and has second-order accuracy in both space and time, based on the so-called central scheme (Kurganov \& Tadmor 2000; Nagakura \& Yamada 2008; Nagakura et al. 2011); spherical coordinates are adopted; and Newtonian selfgravity is taken into account. The Boltzmann solver adopts the discrete-ordinate method, or the $S_{N}$ scheme (Mezzacappa \& Bruenn 1993b; Liebendöfer et al. 2004; Sumiyoshi et al. 2005), finite-differencing both space and momentum space. It is semiimplicit in time, and special relativity is fully accounted for by utilizing two different energy grids: the Lagrangian-remapped and laboratory-fixed grids. Although we normally deploy $12-15$ energy grid points spaced logarithmically between 1 and $300 \mathrm{MeV}$ in this sort of simulation, we increase the number to 


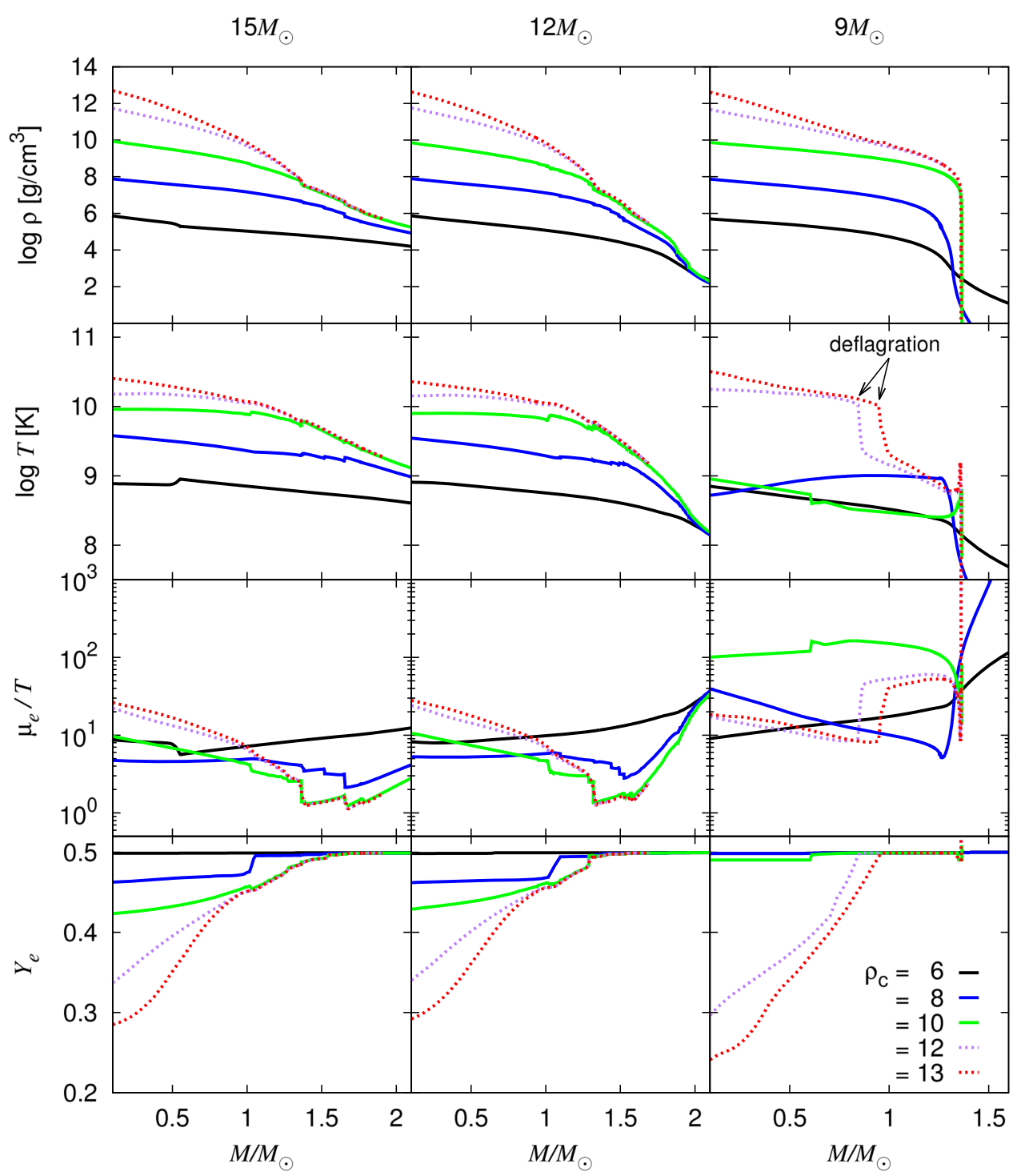

Figure 3. Radial profiles of the density, temperature, degeneracy, and electron fraction at different times. The horizontal axis is the mass coordinate in solar mass units. The left, middle, and right columns correspond to the 15,12 , and $9 M_{\odot}$ models, respectively. Different colors denote the times when the central density becomes $\log _{10} \rho_{c} /\left[\mathrm{g} \mathrm{cm}^{-3}\right]=6$ (black solid), 8 (blue solid), 10 (green solid), 12 (purple dotted), and 13 (red dotted), respectively.

20 , extending at the same time the energy range to lower values, $0.1 \mathrm{MeV}$, in this study so that we could obtain better resolution at these low energies. See Nagakura et al. (2014) for more details.

We use Furusawa's EOS (Furusawa et al. 2013), a multinuclear species EOS, which is based on the relativistic mean field theory with the TM1 parameter set employed in H. Shen's EOS, or the STOS EOS (Shen et al. 2011). It takes into account the NSE among $\sim 8.7 \times 10^{5}$ nuclides and nucleons by extending a nuclear mass formula (Koura et al. 2005; Audi et al. 2012); electron capture rates for heavy nuclei are also provided by this EOS at high densities (see below).

In neutrino transport, the following reactions are taken into account in this paper:

1. Neutrino emissions and absorptions: ECs on nuclei and free nucleons, electron-positron annihilations, nucleonbremsstrahlungs, and their inverse reactions.

2. Neutrino scatterings: isoenergetic scatterings on free nucleons, coherent scatterings on nuclei, and nonisoenergetic scatterings on electrons and positrons.
The reaction rates are based on Bruenn (1985) and Mezzacappa \& Bruenn (1993a), except for the EC on heavy nuclei, for which we take the values provided by Fuller et al. (1985), Oda et al. (1994), Langanke \& Martínez-Pinedo (2001), and Langanke et al. (2003), which we refer to as FFN, ODA, LMP, and LMSH, respectively, and average them over the NSE abundance of the nuclei given by the EOS. We also employ an approximation formula (see Equation (22) below) when none of the tables provides the rate. The luminosity and energy spectrum of $\nu_{e}$ in the collapse phase are obtained directly from the simulations, whereas those for other neutrino species are calculated in the post-processes (see the next section).

For the dynamical simulations, we only use the radial profiles of the central cores derived from the quasi-static evolutions of the progenitors. For the Fe cores of the 12 and $15 M_{\odot}$ models, we start the computations from the time when the central density is $\log _{10} \rho_{c} /\left[\mathrm{g} \mathrm{cm}^{-3}\right]=10.3$. We first run the Boltzmann solver alone with all quantities other than the neutrino distribution functions being fixed until steady states are reached. This step is necessary to avoid artificial 
discontinuities in the neutrino luminosities at the point of switching to dynamical calculations. The $9 M_{\odot}$ model needs special treatment. As already mentioned, gravitational contraction starts in the ONe core via EC. Neon and oxygen are then ignited at the center, and the deflagration wave propagates outward in the core, establishing NSE in its wake. Note that NSE already prevails in the Fe cores. In the case of the $\mathrm{ONe}$ core, we thus have to handle this nuclear equilibration process, solving network equations in addition to the hydrodynamics and neutrino transport. This has been done recently by $\mathrm{K}$. Takahashi et al. (2017, in preparation), and we will use their results in this paper. Since details will be published in their forthcoming paper, we only give here the important information: they modified the 1D radiation-hydrodynamics code developed by Sumiyoshi \& Yamada (2012) to compute nuclear reactions with a network of 40 nuclear species; H. Shen's original EOS is employed instead of Furusawa's extended version; and EC rates are adopted from Juodagalvis et al. (2008). The radial profile at the time when $\log _{10} \rho_{c} /\left[\mathrm{g} \mathrm{cm}^{-3}\right]=10.1$ is used as the initial condition for the simulation.

In all three cases, we terminate the simulations when the central density exceeds $\log _{10} \rho_{c} /\left[\mathrm{g} \mathrm{cm}^{-3}\right]=13$. This is because nuclei become very large thereafter, and pastas are supposed to emerge eventually toward core bounce (Ravenhall et al. 1983). Therefore, the EC rates on these nuclei are highly uncertain and treated only crudely in the original radiationhydrodynamics code.

The dotted lines in Figures 1 and 3 show the evolutions in the collapse phase. The behavior of the central temperature and density in this phase is not much different between the two types of progenitors. In the $9 M_{\odot}$ progenitor, however, the temperature is high only inside the deflagration front, which is located at the mass coordinate of $\sim 1 M_{\odot}$ in Figure 3 . The NSE condition $\left(T \gtrsim 5 \times 10^{9} \mathrm{~K}\right)$ is indeed achieved, and the degeneracy of electrons is partially lifted there. It is also evident that EC is drastically enhanced once NSE is established. We note in passing that the differences in $Y_{e}$ between the $\mathrm{ONe}$ core and $\mathrm{Fe}$ cores presented here may partially reflect the differences in the EOS and EC rates adopted in these models.

\section{Neutrino Emissions}

Neutrinos are emitted via several processes, which are classified into thermal pair emissions and nuclear weak interactions. In this section, we first describe in some detail the formulae we employ to evaluate the neutrino emissivity for individual processes (Sections 3.1-3.3). In order to discuss the possibility of observations at terrestrial detectors, flavor oscillations should be taken into account and will be discussed in Section 3.4. In the progenitor phase, we simply evaluate the luminosities and spectra of all flavors of neutrinos in postprocesses, i.e., we extract the density, temperature, and electron fraction profiles from the data obtained in the stellar evolution calculations and core-collapse simulations at appropriate times from $\sim 10^{6} \mathrm{~s}$ to a few $\mathrm{ms}$ before core bounce. Then we calculate pointwise the neutrino emissivities for the thermal pair productions and nuclear weak interactions (see Table 1), and integrate the results outwards from the center of the star until the number luminosities do not change appreciably, $\sim 10^{-6} \%$.

In the collapse phase, on the other hand, we treat $\nu_{e}$ differently from the other flavors of neutrinos: the luminosity and spectrum of $\nu_{e}$ are derived directly from the radiation-hydrodynamical simulations since we have to take into account neutrino transport when the opacity for neutrinos gets high enough to hinder their free escape. Note that $\nu_{e}$ are responsible for the transfer of the electron-type lepton number and hence the evolution of $Y_{e}$, and their transport in the core is indispensable for realistic supernova simulations.

Other species of neutrinos in the collapse phase, on the other hand, are treated in post-processes, i.e., we first run simulations neglecting these reactions; ${ }^{9}$ we then extract the densities, temperatures, and electron fractions as well as the distribution function of $\nu_{e}$ from the results of the simulations and plug them into the formulae of emissivities given in the following subsections. Note that the distribution function of $\nu_{e}$ is necessary to take into account the Fermi-blocking in the final state. We ignore the transport of these neutrinos, since they are much less abundant than $\nu_{e}$. In fact, we compare the emissivities of $\bar{\nu}_{e}$ via $\beta^{-}$decay inside the opaque part of the core $\left(\log _{10} \rho /\left[\mathrm{g} / \mathrm{cm}^{3}\right]>11.5\right)$ with those in entire NSE regions when the central density is $\log _{10} \rho_{c} /\left[\mathrm{g} / \mathrm{cm}^{3}\right]=13$ and find that the former contributes only $\sim 0.001 \%$ to the total neutrino emissivities because of the high degeneracy of the electrons there. The emissions of these neutrinos after matter becomes opaque are hence negligible compared with those before that. The neutrino emission processes and their treatments in our calculations are summarized in Table 1.

\subsection{Thermal Emissions of Neutrino Pairs}

For this type of emission, we normally consider four processes: electron-positron annihilation, plasmon decay, bremsstrahlung, ${ }^{10}$ and photo processes. They produce all flavors of neutrinos. The reaction rates of these processes depend mainly on three hydrodynamical variables: density, temperature, and electron fraction (or electron chemical potential).

Itoh et al. (1996) investigated in detail which process is dominant in which regime. In Paper I, we found that electronpositron pair annihilation is always dominant for FeCCSN progenitors with 12 and $15 M_{\odot}$, while for the ONeCCSN progenitor with $9 M_{\odot}$, plasmon decay prevails until $\mathrm{Ne}$ and $\mathrm{O}$ are ignited at the center and the temperature rises quickly, after which pair annihilation takes over. In this paper, we hence focus on these two processes as in Paper I. See also Patton et al. (2017) and Guo \& Qian (2016).

\subsubsection{Electron-Positron Annihilation}

Neutrino-pair creations through electron-positron annihilations become important at high temperatures $\gtrsim 10^{9} \mathrm{~K}$ simply because the number of photons with high enough energies to produce electron-positron pairs becomes large, and as a result, electron-positron pairs also become abundant at these temperatures. Detailed derivations of the reaction rate $R$ for pair annihilation are given in Paper I (Appendix A.1) but with some typographical errors. We give here the correct expression for $R$

\footnotetext{
9 The productions and absorptions of neutrinos via electron-positron pair annihilations are included in the simulations of core collapse. The resolution of the energy spectra obtained in the simulations is rather low and rough, and we re-construct them in the post-process.

${ }^{10}$ This bremmsstrahlung occurs in association with a collision of an electron with a nucleus via electromagnetic interactions and is different from the bremsstrahlung from nucleon-nucleon collisions via nuclear forces, which becomes important in the post-bounce phase.
} 
Table 1

Neutrino Reactions Considered in this Paper

\begin{tabular}{|c|c|c|c|c|}
\hline & & Reactions & Collapse Phase ${ }^{\mathrm{a}}$ & Colors $^{\mathrm{b}}$ \\
\hline Thermal Processes & $\begin{array}{c}\text { Pair } \\
\text { Plasmon }\end{array}$ & $\begin{array}{l}e^{-}+e^{+} \longrightarrow \nu+\bar{\nu} \\
\gamma^{*} \longrightarrow \nu+\bar{\nu}\end{array}$ & $\begin{array}{c}\nu_{e}: \mathrm{T}, \text { Others: } \mathrm{P} \\
\ldots\end{array}$ & $\begin{array}{l}\text { Red } \\
\text { Brown }\end{array}$ \\
\hline
\end{tabular}

Notes.

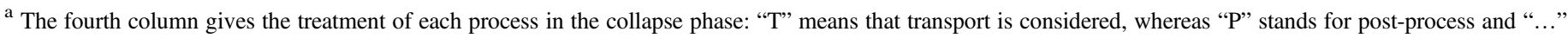
implies that the process is neglected.

b The fifth column lists the color codes used in Figures 5-9.

(Mezzacappa \& Bruenn 1993a; Schinder \& Shapiro 1982):

$$
R=\frac{8 G_{F}^{2}}{(2 \pi)^{2}}\left[\beta_{1} I_{1}+\beta_{2} I_{2}+\beta_{3} I_{3}\right]
$$

In this expression, the $\beta$ are the following combinations of coupling constants: $\beta_{1}=\left(C_{V}-C_{A}\right)^{2}, \beta_{2}=\left(C_{V}+C_{A}\right)^{2}$, and $\beta_{3}=C_{V}^{2}-C_{A}^{2}$, and the $I$ are the functions of the energies of the emitted neutrino $E_{\nu}$ and anti-neutrino $E_{\bar{\nu}}$ and the angle $\theta$ between their momenta $\boldsymbol{q}$ and $\boldsymbol{q}^{\prime}$ :

$$
\begin{aligned}
I_{1}\left(E_{\nu},\right. & \left.E_{\bar{\nu}}, \cos \theta\right) \\
=- & \frac{2 \pi T E_{\nu}^{2} E_{\bar{\nu}}^{2}(1-\cos \theta)^{2}}{\left[\exp \left(\frac{E_{\nu}+E_{\bar{\nu}}}{T}\right)-1\right] \Delta_{e}^{5}} \\
\times & \left\{A T ^ { 2 } \left(\left[G_{2}\left(y_{\max }\right)-G_{2}\left(y_{\min }\right)\right]\right.\right. \\
+ & {\left[2 y_{\max } G_{1}\left(y_{\max }\right)-2 y_{\min } G_{1}\left(y_{\min }\right)\right] } \\
+ & {\left.\left[y_{\max }^{2} G_{0}\left(y_{\max }\right)-y_{\min }^{2} G_{0}\left(y_{\min }\right)\right]\right) } \\
+ & B T\left(\left[G_{1}\left(y_{\max }\right)-G_{1}\left(y_{\min }\right)\right]\right. \\
+ & {\left.\left[y_{\max } G_{0}\left(y_{\max }\right)-y_{\min } G_{0}\left(y_{\min }\right)\right]\right) } \\
+ & \left.C\left[G_{0}\left(y_{\max }\right)-G_{0}\left(y_{\min }\right)\right]\right\}, \\
& I_{2}=I_{1}\left(E_{\bar{\nu}}, E_{\nu}, \cos \theta\right), \\
I_{3}= & -\frac{2 \pi T m_{e}^{2} E_{\nu} E_{\bar{\nu}}(1-\cos \theta)}{\left[\exp \left(\frac{E_{\nu}+E_{\bar{\nu}}}{T}\right)-1\right] \Delta_{e}} \\
& \times\left[G_{0}\left(y_{\max }\right)-G_{0}\left(y_{\min }\right)\right],
\end{aligned}
$$

with

$$
\begin{gathered}
\Delta_{e}{ }^{2} \equiv E_{\bar{\nu}}^{2}+E_{\nu}^{2}+2 E_{\nu} E_{\bar{\nu}} \cos \theta, \\
A=E_{\bar{\nu}}^{2}+E_{\nu}{ }^{2}-E_{\nu} E_{\bar{\nu}}(3+\cos \theta), \\
B=\left[-2 E_{\nu}{ }^{2}+E_{\bar{\nu}}^{2}(1+3 \cos \theta)\right. \\
\left.\quad+E_{\nu} E_{\bar{\nu}}(3-\cos \theta)\right] E_{\nu}, \\
C=\left[\left(E_{\nu}+E_{\bar{\nu}} \cos \theta\right)^{2}-\frac{1}{2} E_{\bar{\nu}}^{2}\left(1-\cos ^{2} \theta\right)\right. \\
\left.-\frac{1}{2}\left(\frac{m_{e} \Delta_{e}}{E_{\nu}}\right)^{2} \frac{1+\cos \theta}{1-\cos \theta}\right] E_{\nu}{ }^{2},
\end{gathered}
$$

and $\quad \eta^{\prime}=\left(\mu_{e}+E_{\nu}+E_{\bar{\nu}}\right) / T, \quad \eta=\mu_{e} / T, y_{\max }=E_{\max } / T$, $y_{\text {min }}=E_{\min } / T$, and $G_{n}(y) \equiv F_{n}\left(\eta^{\prime}-y\right)-F_{n}(\eta-y)$, in which the Fermi integral $F_{n}(z)$ is defined as

$$
F_{n}(z)=\int_{0}^{\infty} \frac{x^{n}}{e^{x-z}+1} d x
$$

The differential number emissivity for neutrinos or antineutrinos, $d Q_{N}^{\nu_{i}} / d E_{\nu}$, in the progenitor phase is simply given as an integral of $R$ over the momentum of the partner. We employ spherical coordinates in momentum space (See Paper I for details). The energy integral was then truncated at some maximum values, which are determined empirically from the temperature. Our results are in good agreement with those derived with the Monte Carlo method in Yoshida et al. (2016) within errors of $4.5 \%$.

In the collapse phase, as the matter density increases and the neutrino energy rises, interactions between matter and neutrinos can no longer be ignored. Electron-type neutrinos, the most abundant species, are eventually trapped in the core at $\log _{10} \rho /\left[\mathrm{g} \mathrm{cm}^{-3}\right] \gtrsim 11$ and become degenerate. Then, the pair creation of $\nu_{e}$ and $\bar{\nu}_{e}$ is suppressed by Fermi-blocking in the final state. Considering the inverse process, we should hence modify the differential emissivity of $\bar{\nu}_{e}$ in this phase as

$$
\begin{aligned}
& \frac{d Q_{N}^{\bar{\nu}_{e}}}{d E_{\bar{\nu}_{e}} d \cos \theta_{\bar{\nu}_{e}} d \phi_{\bar{\nu}_{e}}} \\
& =\frac{E_{\bar{\nu}_{e}}}{2(2 \pi)^{3}} \int \frac{d^{3} q_{\nu_{e}}}{(2 \pi)^{3} 2 E_{\nu_{e}}} \\
& \times\left[R^{p}\left(E_{\nu_{e}}, E_{\bar{\nu}_{e}}, \cos \theta\right)\left[1-f_{\nu_{e}}\left(E_{\nu_{e}}, \theta_{\nu_{e}}\right)\right]\right. \\
& \times\left[1-f_{\bar{\nu}_{e}}\left(E_{\bar{\nu}_{e}}, \theta_{\bar{\nu}_{e}}\right)\right] \\
& \left.-R^{a}\left(E_{\nu_{e}}, E_{\bar{\nu}_{e}}, \cos \theta\right) f_{\nu_{e}}\left(E_{\nu_{e}}, \theta_{\nu_{e}}\right) f_{\bar{\nu}_{e}}\left(E_{\bar{\nu}_{e}}, \theta_{\bar{\nu}_{e}}\right)\right] \text {, }
\end{aligned}
$$

where $f_{\nu_{e}}$ and $f_{\bar{\nu}_{e}}$ are the distribution functions of $\nu_{e}$ and $\bar{\nu}_{e}$, respectively. The direction of neutrino momentum is specified with the zenith and azimuth angles $\left(\theta_{\nu}, \phi_{\nu}\right)$ with respect to the local radial direction. The first term in the integrand on the right-hand side is the production rate whereas the second term represents the absorption rate for the inverse reaction: $R_{p}$ is given by Equation (1) while $R_{a}$ is obtained from $R_{p}$ via the detailed balance condition $R_{a}=R_{p} \exp \left(\left(E_{\nu}+E_{\bar{\nu}}\right) / T\right)$. We make an approximation $1-f_{\bar{\nu}_{e}}\left(E_{\bar{v}_{e}}, \theta_{\bar{v}_{e}}\right) \sim 1$, which is well justified as $f_{\bar{\nu}_{e}}\left(E_{\bar{\nu}_{e}}, \theta_{\bar{\nu}_{e}}\right)$ is small in the collapse phase.

Moreover, we have to take into account matter motions in the collapse phase and distinguish the global inertial frame, or the observer's frame, from the local fluid-rest frame, since the 
emissivities we have presented so far are all valid in the latter frame. The emissivities in the observer's frame is obtained using the following transformation,

$$
\frac{d Q_{N}^{\bar{\nu}_{e}}}{d E_{\bar{\nu}_{e}}^{\mathrm{lab}} d \cos \theta_{\bar{\nu}_{e}}^{\mathrm{lab}} d \phi_{\bar{\nu}_{e}}^{\mathrm{lab}}}=J \frac{d Q_{N}^{\bar{\nu}_{e}}}{d E_{\bar{\nu}_{e}}^{\mathrm{fr}} d \cos \theta_{\bar{\nu}_{e}}^{\mathrm{fr}} d \phi_{\bar{\nu}_{e}}^{\mathrm{fr}}},
$$

where the superscripts "lab" and "fr" stand for quantities in the laboratory and fluid-rest frames, respectively, and $J$ is the Jacobian

$$
J=\frac{\partial\left(E_{\bar{\nu}_{e}}^{\mathrm{fr}}, \cos \theta_{\bar{\nu}_{e}}^{\mathrm{fr}}, \phi_{\bar{\nu}_{e}}^{\mathrm{fr}}\right)}{\partial\left(E_{\bar{\nu}_{e}}^{\mathrm{lab}}, \cos \theta_{\bar{\nu}_{e}}^{\mathrm{lab}}, \phi_{\bar{\nu}_{e}}^{\mathrm{lab}}\right)}
$$

for the following transformations:

$$
\begin{gathered}
E^{\mathrm{fr}}=E^{\mathrm{lab}} \gamma\left(1-\boldsymbol{n}^{\mathrm{lab}} \cdot \boldsymbol{v}\right), \\
\boldsymbol{n}^{\mathrm{fr}}=\frac{1}{\gamma\left(1-\boldsymbol{n}^{\mathrm{lab}}\right)}\left[\boldsymbol{n}^{\mathrm{lab}}+\left(-\gamma+\frac{\gamma-1}{v^{2}} \boldsymbol{v} \cdot \boldsymbol{n}^{\mathrm{lab}}\right)\right],
\end{gathered}
$$

with $\boldsymbol{n}^{\text {lab }}=\left(\sin \theta_{\nu} \cos \phi_{\nu}, \sin \theta_{\nu} \sin \phi_{\nu}, \cos \theta_{\nu}\right)$ being the propagation direction of neutrinos.

\subsubsection{Plasmon Decay}

Plasmon decay is one of the main cooling processes in massive stars after $\mathrm{C}$ burning. As we explained already, it is the dominant neutrino-emitting reaction in the ONe core until NSE is established.

Although the reaction rates for the plasmon decay were given in Paper I (Appendix A.2), we give the expression for $R$ for completeness (Braaten \& Seagel 1993):

$$
\begin{aligned}
R= & \left(\frac{G_{F}}{\sqrt{2}}\right)^{2} \frac{16 C_{V}^{2}}{e^{2}} \frac{2 E_{\nu}^{2} E_{\bar{\nu}}^{2}(1-\cos \theta)}{\left[1-\exp \left(\frac{E_{\nu}+E_{\bar{\nu}}}{T}\right)\right]} \\
& \times\left\{\frac{3 \omega_{p}^{2}}{\Delta_{e}^{2}} \delta\left(f_{L}\left(E_{\nu}, E_{\bar{\nu}}, \cos \theta\right)\right)\right. \\
& \times\left[\frac{E_{\nu}+E_{\bar{\nu}}}{2 \Delta_{e}} \ln \frac{E_{\nu}+E_{\bar{\nu}}-\Delta_{e}}{E_{\nu}+E_{\bar{\nu}}+\Delta_{e}}+1\right] \\
& \times\left[-2 \cos \theta\left(E_{\nu}+E_{\bar{\nu}}\right)^{2}-2 E_{\nu} E_{\bar{\nu}} \sin ^{2} \theta\right. \\
& \left.+\frac{2\left(E_{\nu}+E_{\bar{\nu}}\right)^{2}}{\Delta_{e}^{2}}\left(E_{\nu}+E_{\bar{\nu}} \cos \theta\right)\left(E_{\bar{\nu}}+E_{\nu} \cos \theta\right)\right] \\
& -\frac{3 \omega_{p}^{2}\left(E_{\nu}+E_{\bar{\nu}}\right)^{2}}{\Delta_{e}^{2}} \delta\left(f_{T}\left(E_{\nu}, E_{\bar{\nu}}, \cos \theta\right)\right) \\
& \times\left[1+\frac{E_{\nu} E_{\bar{\nu}}(1-\cos \theta)}{\left(E_{\nu}+E_{\bar{\nu}}\right) \Delta_{e}} \ln \frac{E_{\nu}+E_{\bar{\nu}}-\Delta_{e}}{E_{\nu}+E_{\bar{\nu}}+\Delta_{e}}\right] \\
& \left.\times\left[1-\frac{\left(E_{\nu} \cos \theta+E_{\bar{\nu}}\right)\left(E_{\bar{\nu}} \cos \theta+E_{\nu}\right)}{\Delta_{e}^{2}}\right]\right\},
\end{aligned}
$$

with the following definitions of $f_{L}\left(E_{\nu}, E_{\bar{\nu}}, \cos \theta\right)$ and $f_{T}\left(E_{\nu}, E_{\bar{\nu}}, \cos \theta\right)$ :

$$
\begin{aligned}
& f_{L}\left(E_{\nu}, E_{\bar{\nu}}, \cos \theta\right)=2 E_{\nu} E_{\bar{\nu}}(1-\cos \theta) \\
& \quad+\frac{6 \omega_{p}^{2} E_{\nu} E_{\bar{\nu}}(1-\cos \theta)}{\Delta_{e}^{2}}\left[\frac{E_{\nu}+E_{\bar{\nu}}}{2 \Delta_{\nu}} \ln \frac{E_{\nu}+E_{\bar{\nu}}-\Delta_{e}}{E_{\nu}+E_{\bar{\nu}}+\Delta_{e}}+1\right]
\end{aligned}
$$

$$
\begin{aligned}
f_{T}\left(E_{\nu}, E_{\bar{\nu}}, \cos \theta\right) \\
=2 E_{\nu} E_{\bar{\nu}}(1-\cos \theta) \\
\quad-\frac{3 \omega_{p}^{2}\left(E_{\nu}+E_{\bar{\nu}}\right)^{2}}{2 \Delta_{e}^{2}} \\
\quad \times\left[1+\frac{E_{\nu} E_{\bar{\nu}}(1-\cos \theta)}{\left(E_{\nu}+E_{\bar{\nu}}\right) \Delta_{e}} \ln \frac{E_{\nu}+E_{\bar{\nu}}-\Delta_{e}}{E_{\nu}+E_{\bar{\nu}}+\Delta_{e}}\right] .
\end{aligned}
$$

Note that the dispersion relations of the longitudinal and transverse modes are obtained from $f_{L}=0$ and $f_{T}=0$, respectively.

The differential and total emissivities are defined in the same way as for the pair annihilation. The Dirac deltas in Equation (15) are used in the angular integral. Note that neutrinos emitted by plasmon decay have low energies, $E_{\nu} \sim 0.5 \mathrm{MeV}$, and their contribution to the observable luminosity is minor even in the pre-collapse phase (see Paper I for details). This is even more so in the collapse phase. We hence estimate only the maximum luminosities for the plasmon decay in the collapse phase, ignoring the Fermi-blocking in the final state.

\subsection{Nuclear Weak Interactions}

This is the new material in this paper, which was ignored in Paper I. In the late evolutionary phase of progenitors and during the collapse phase, nuclear weak interactions can no longer be ignored. In particular, once opened, ECs by heavy nuclei become the dominant reactions. They play an important role in the hydrodynamics of core collapse as explained earlier. Although $\beta^{+}$decays of heavy nuclei also emit $\nu_{e}$, they are certainly is subdominant. $\bar{\nu}_{e}$ are emitted either by positron captures (PCs) or $\beta^{-}$decays. Although they never affect the core dynamics up to bounce, they are important from the observational point of view, since it is mainly water Cherenkov detectors that observe them. Moreover, Patton et al. (2017) pointed out that there may be a period in which $\beta^{-}$decay dominates pair annihilation in the production of $\bar{\nu}_{e}$.

In this paper, we thus take into account the following reactions:

1. electron capture (EC)

$$
(Z, A)+e^{-} \longrightarrow(Z-1, A)+\nu_{e}
$$

2. $\beta^{+}$decay

$$
(Z, A) \longrightarrow(Z-1, A)+e^{+}+\nu_{e}
$$

3. positron capture $(\mathrm{PC})$

$$
(Z, A)+e^{+} \longrightarrow(Z+1, A)+\bar{\nu}_{e}
$$

4. $\beta^{-}$decay

$$
(Z, A) \longrightarrow(Z+1, A)+e^{-}+\bar{\nu}_{e}
$$

In the above expressions, $Z$ and $A$ are the atomic and mass numbers of nuclei, respectively. We consider in this paper 17,502 nuclei $(6<Z<160,2<N<320)$ for EC and 3928 nuclei $(7<Z<117,9<N<200)$ for $\beta^{-}$decay (see also Figure 4).

For the calculations of the luminosities and energy spectra of neutrinos, we use the FFN, ODA, LMP, and LMSH tables whenever available. They normally give us the total reaction rates and average neutrino energies. If more than one table is 
EC

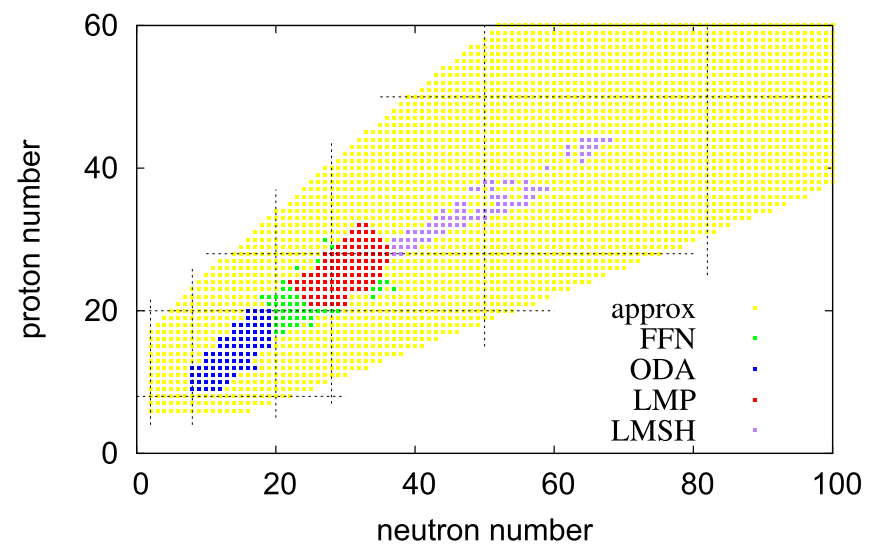

$\beta^{-}$

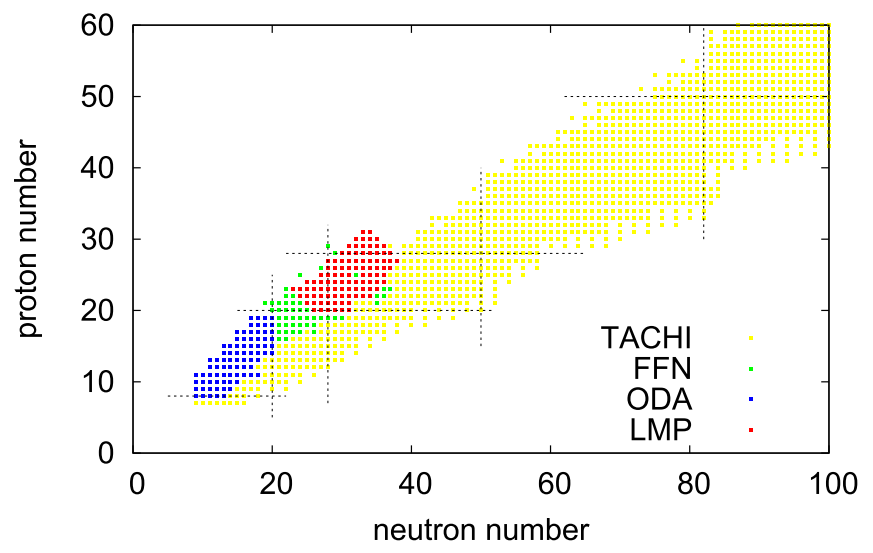

Figure 4. Nuclear charts indicating in different colors the nuclear species with the reaction rates for EC (left) and $\beta^{-}$decay (right) given in the LMSH (black; Langanke et al. 2003), LMP (red; Langanke \& Martínez-Pinedo 2001), ODA (blue; Oda et al. 1994), FFN (green; Fuller et al. 1985), and TACHI (yellow; Tachibana \& Yamada 1995; Tachibana 2000; Yoshida \& Tachibana 2000; Koura et al. 2003; Koura 2004; Koura et al. 2005) tables, as well as using the approximation formula (yellow; Equation (22)).

available for the same nucleus, we adopt one of them in the following order: $\mathrm{LMSH}>\mathrm{LMP}>\mathrm{ODA}>$ FFN. Note that the LMSH table only includes data on the $\nu_{e}$ emission via EC. If no information is available from these tables, which actually happens when very heavy and/or neutron-rich nuclei $(A, N)$ become populated at late times in the collapse phase, we employ the approximation formulae for $Q_{N \text {,EC }}$ and $Q_{E \text {, EC }}$ (Fuller et al. 1985; Langanke et al. 2003; Sullivan et al. 2016):

$$
\begin{aligned}
Q_{N, \mathrm{EC}}^{\nu_{e}}= & \sum_{i} \frac{X_{i} \rho}{m_{p} A_{i}} \frac{\ln 2 \cdot B}{K}\left(\frac{T}{m_{e} c^{2}}\right)^{5} \\
& \times\left[F_{4}(\eta)-2 \chi F_{3}(\eta)+\chi^{2} F_{2}(\eta)\right], \\
Q_{E, \mathrm{EC}}^{\nu_{e}}= & \sum_{i} \frac{X_{i} \rho}{m_{p} A_{i}} \frac{\ln 2 \cdot B}{K}\left(\frac{T}{m_{e} c^{2}}\right)^{6} \\
& \times\left[F_{5}(\eta)-2 \chi F_{4}(\eta)+\chi^{2} F_{3}(\eta)\right],
\end{aligned}
$$

where $K=6146 \mathrm{~s}, \chi=(Q-\Delta E) / T, \eta=\left(\mu_{e}+Q-\Delta E\right) / T$; and $X_{i}$ and $A_{i}$ are the mass fraction and mass number of the nuclear species $i$, respectively. The representative values of the matrix element and the energy level difference between the parent and daughter nuclei are set to $B=4.6$ and
$\Delta E=E_{f}-E_{i}=2.5 \mathrm{MeV}$, respectively, following Langanke et al. (2003). For $\beta^{-}$decay in the absence of data, we consult another table compiled by Tachibana (Tachibana \& Yamada 1995; Tachibana 2000; Yoshida \& Tachibana 2000; Koura et al. 2003; Koura 2004; Koura et al. 2005). Note that the data in this table were theoretically calculated for the terrestrial environment and hence do not take into account the Fermi-blocking of electrons in the final state. We hence re-incorporated them, albeit crudely, in the reaction rates as a suppression factor $1-f_{e}\left(\left\langle E_{e}\right\rangle\right)$ based on the average electron energy $\left\langle E_{e}\right\rangle$, which is given in the Tachibana table. In Figure 4, we summarize which tables or whether the approximation formula is used in which region in the nuclear chart.

The energy spectrum is reconstructed for each reaction by using the effective $q$-value method (Langanke et al. 2001b; Kunugise \& Iwamoto 2007; Patton et al. 2017):

$$
\begin{aligned}
\frac{d Q_{N, k}^{\nu_{j}}}{d E_{\nu_{j}}}= & N_{k} \frac{E_{\nu_{j}}^{2}\left(E_{\nu_{j}}-q\right)^{2}}{1+\exp \left(\frac{E_{\nu_{j}}-q-\mu_{e}}{T}\right)} \\
& \times \Theta\left(E_{\nu_{j}}-q-m_{e}\right),
\end{aligned}
$$

for $k=\mathrm{EC}, \mathrm{PC}$, and

$$
\begin{aligned}
\frac{d Q_{N, k}^{\nu_{j}}}{d E_{\nu_{j}}}= & N_{k} \frac{E_{\nu_{j}}^{2}\left(q-E_{\nu_{j}}\right)^{2}}{1+\exp \left(\frac{E_{\nu_{j}}-q+\mu_{e}}{T}\right)} \\
& \times \Theta\left(q-m_{e}-E_{\nu_{j}}\right),
\end{aligned}
$$

for $k=\beta^{-}, \beta^{+}$, where $\nu_{j}=\nu_{e}$ or $\bar{\nu}_{e}$, and the normalization factor $N_{k}$ is determined by the following relation:

$$
Q_{N, k}=\int \frac{d Q_{N, k}^{\nu_{j}}}{d E_{\nu_{j}}} d E_{\nu_{j}}
$$

The effective $q$-value is actually given from the average energy $\left\langle E_{\nu_{e}}\right\rangle$ as follows:

$$
\begin{aligned}
\frac{Q_{E, \mathrm{EC}}^{\nu_{e}}+Q_{E, \beta^{+}}^{\nu_{e}}}{\lambda_{\mathrm{EC}}+\lambda_{\beta^{+}}} & =\left\langle E_{\nu_{e}}\right\rangle \\
& =\frac{\int E_{\nu_{e}} \frac{d Q_{N}^{\nu_{e}}}{d E_{\nu_{e}}}\left(E_{\nu_{e}}\right) d E_{\nu_{e}}}{\int \frac{d Q_{N}^{\nu_{e}}}{d E_{\nu_{e}}}\left(E_{\nu_{e}}\right) d E_{\nu_{e}}},
\end{aligned}
$$

where the following notation is used:

$$
\frac{d Q_{N}^{\nu_{e}}}{d E_{\nu_{e}}}\left(E_{\nu_{e}}\right)=\frac{d Q_{N, \mathrm{EC}}^{\nu_{e}}}{d E_{\nu_{e}}}+\frac{d Q_{N, \beta^{+}}^{\nu_{e}}}{d E_{\nu_{e}}} .
$$

For $\bar{\nu}_{e}$, we replace the subscripts of $\mathrm{EC}$ and $\beta^{+}$with PC and $\beta^{-}$.

\subsection{Electron Captures on Free Protons}

In the collapse phase, although they are not abundant, ECs on free protons,

$$
p+e^{-} \longrightarrow n+\nu_{e}
$$

cannot be ignored, since the cross-section is larger than that of ECs on heavy nuclei. 
The reaction rate is given by Bruenn (1985) as

$$
\begin{aligned}
\frac{d Q_{N, \mathrm{p}}^{\nu_{e}}}{d E_{\nu_{e}}}= & \frac{G_{F}^{2}}{\pi} \eta_{\mathrm{pn}}\left(g_{V}^{2}+3 g_{A}{ }^{2}\right)\left(E_{\nu_{e}}+Q\right)^{2} \\
& \times \sqrt{1-\frac{m_{e}^{2}}{\left(E_{\nu_{e}}+Q\right)^{2}}} f_{e}\left(E_{\nu_{e}}+Q\right),
\end{aligned}
$$

where the mass difference between neutron and proton is given as $Q=m_{n}-m_{p}$, and the form factors for the vector and axial vector currents are given as $g_{V}=1$ and $g_{A}=1.23$, respectively; $\eta_{\mathrm{pn}}$ is defined as

$$
\begin{aligned}
\eta_{\mathrm{pn}} & \equiv \int \frac{2 d^{3} p}{(2 \pi)^{3}} \tilde{F}_{p}(\tilde{E})\left[1-\tilde{F}_{n}(\tilde{E})\right] \\
& =\frac{n_{n}-n_{p}}{\exp \left(\frac{\mu_{n}^{0}-\mu_{p}^{0}}{T}\right)-1} .
\end{aligned}
$$

In the above expression, the Fermi-Dirac distributions are denoted by $\tilde{F}_{i}(\tilde{E})=1 /\left[1+\exp \left(\tilde{E}-\mu_{i}\right) / T\right](i=p, n)$, and the number densities and chemical potentials not including the rest-mass energies of proton and neutron are written as $n_{n}, n_{p}$ and $\mu_{p}^{0}, \mu_{n}^{0}$, respectively; the non-relativistic expression $\tilde{E} \sim p_{i}^{2} / 2 m$ is employed for the kinetic energies of nucleons.

In our calculations, the $\mathrm{PC}$ and $\beta^{-}$decay on neutrons were ignored because they make very little contributions. This is simply because free neutrons are scarce. In addition, the $\beta^{-}$ decay of free neutron is severely suppressed by the Fermiblocking of electrons in the final state. Note also that the energy of neutrinos emitted by free neutrons is lower than that emitted by nuclei.

\subsection{Neutrino Oscillations}

The electron neutrinos and anti-neutrinos may convert to $\nu_{x}$ and $\bar{\nu}_{x}$, respectively, and vice versa during propagation as a result of flavor oscillations. We only take into account the vacuum oscillations and MSW effect and ignore the collective oscillations, which will probably not occur in the pre-bounce phase. The so-called survival probabilities of $\nu_{e}$ and $\bar{\nu}_{e}$, denoted by $p$ and $p^{\prime}$, respectively, are given in the adiabatic limit as

$$
\begin{aligned}
& p= \begin{cases}\sin ^{2} \theta_{13}=0.0234 & \text { for normal hierarchy, } \\
\sin ^{2} \theta_{12} \cos ^{2} \theta_{13}=0.300 & \text { for inverted hierarchy, }\end{cases} \\
& p^{\prime}= \begin{cases}\cos ^{2} \theta_{12} \cos ^{2} \theta_{13}=0.676 & \text { for normal hierarchy, } \\
\sin ^{2} \theta_{13}=0.0234 & \text { for inverted hierarchy, }\end{cases}
\end{aligned}
$$

with $\cos ^{2} \theta_{12}=0.692, \cos ^{2} \theta_{13}=0.977$ (Particle Data Group 2014). The definition of the mixing angles is common and given in Paper I.

Since stars are not homogeneous, we need to calculate the number and energy emissivities per volume and time, $Q_{N}^{\nu}$ and $Q_{E}^{\nu}$, as well as the spectra, $d Q_{N}^{\nu} / d E_{\nu}$, as a function of radius, and integrate them over the star to obtain the number and energy luminosities, $L_{N}^{\nu}$ and $L_{E}^{\nu}$, together with the observed spectra, $d L_{N}^{\nu} / d E_{\nu}$, for all flavors of neutrinos in the progenitor phase and for neutrinos other than $\nu_{e}$ in the collapse phase. We take the stellar radius as the upper limit of the integrals in principle, although the integration that was started from the center is terminated at some radius where the value no longer changes appreciably. For the nuclear weak processes, we take the upper limit as the radius of the NSE region. We evaluate the above quantities at different times so that their time evolutions could be obtained.

As for the pair processes in the collapse phase, we need to actually conduct two more integrations concerning the zenith and azimuth angles (see Equation (10)). In doing so, we distinguish the observer's frame from the local fluid-rest frame in the collapse phase (see Equation (11)). Then, the differential and total number luminosities are given as follows:

$$
\begin{aligned}
& \frac{d L_{N}^{\bar{\nu}_{e}}}{d E_{\bar{\nu}_{e}}^{\mathrm{lab}}}=\left.\int \frac{d Q_{N}^{\bar{\nu}_{e}}}{d E_{\bar{\nu}_{e}}^{\mathrm{lab}} d \cos \theta_{\bar{\nu}_{e}}^{\mathrm{lab}} d \phi_{\bar{\nu}_{e}}^{\mathrm{lab}}}\right|_{\theta_{\bar{\nu}_{e}}^{\mathrm{lab}}=\theta_{s}, \phi_{\bar{\nu}_{e}}^{\mathrm{lab}}=180^{\circ}} d V \\
&=\left.\int \frac{d Q_{N}^{\bar{\nu}_{e}}}{d E_{\bar{\nu}_{e}}^{\mathrm{lab}} d \cos \theta_{\bar{\nu}_{e}}^{\mathrm{lab}} d \phi_{\bar{\nu}_{e}}^{\mathrm{lab}}}\right|_{\theta_{\bar{\nu}_{e}}^{\mathrm{lab}}=\theta_{s}, \phi_{\bar{\nu}_{e}}^{\mathrm{lab}}=180^{\circ}} \\
& \times 2 \pi r^{2} d r d \cos \theta_{s}, \\
& L_{N}^{\bar{\nu}_{e}}=\int \frac{d L_{N}^{\bar{\nu}_{e}}}{d E_{\bar{\nu}_{e}}^{\mathrm{lab}}} d E_{\bar{\nu}_{e}}^{\mathrm{lab}} .
\end{aligned}
$$

In writing these expressions, we assume that the observer is located at infinity on the positive $z$-axis. Note that we employ these formulae only for electron-positron annihilation, since it dominates over plasmon decay in the collapse phase. As for the latter, we only give a rough estimate, ignoring the frame difference and the Fermi-blocking in the final state.

The differential luminosities, or energy spectra, of $\nu_{e}$ and $\bar{\nu}_{e}$ with the vacuum and MSW neutrino oscillations that are being taken into account in the adiabatic limit are given as follows:

$$
\begin{aligned}
& \left(\frac{d L_{N}^{\nu_{e}}}{d E_{\nu_{e}}}\right)_{\mathrm{osc}}=p\left(\frac{d L_{N}^{\nu_{e}}}{d E_{\nu_{e}}}\right)_{0}+(1-p)\left(\frac{d L_{N}^{\nu_{x}}}{d E_{\nu_{x}}}\right)_{0}, \\
& \left(\frac{d L_{N}^{\bar{\nu}_{e}}}{d E_{\bar{\nu}_{e}}}\right)_{\mathrm{osc}}=p^{\prime}\left(\frac{d L_{N}^{\bar{\nu}_{e}}}{d E_{\bar{\nu}_{e}}}\right)_{0}+\left(1-p^{\prime}\right)\left(\frac{d L_{N}^{\bar{\nu}_{x}}}{d E_{\bar{\nu}_{x}}}\right)_{0} .
\end{aligned}
$$

In these expressions, the subscript " 0 " means that the original spectra before neutrino oscillations are considered; $\nu_{x}$ stands for $\nu_{\mu}$ or $\nu_{\tau}$, both of which we assume are produced solely by electron-positron pair annihilations and have the same spectrum.

\section{Results}

In the following, we present the main results: the number luminosities as well as the energy spectra for different neutrino flavors as functions of time. Based on them, we then estimate the expected numbers of detection events for different terrestrial neutrino detectors.

\subsection{Luminosity and Spectrum}

In Figure 5, we show the time evolutions of the number luminosities of $\nu_{e}$ and $\bar{\nu}_{e}$ for the $15 M_{\odot}$ progenitor model. The left and right panels display the progenitor and collapse phases, respectively. The origin of the time coordinate corresponds to the time the hydrodynamical calculations are initiated. The solid and dashed lines denote $\bar{\nu}_{e}$ and $\nu_{e}$, respectively. The colors of the lines indicate the contributions from different processes 


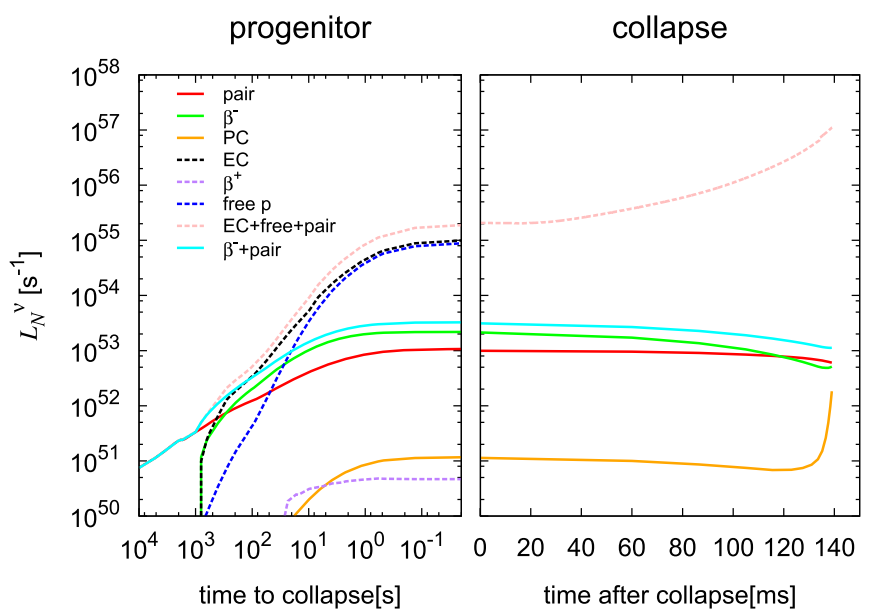

Figure 5. Time evolution of the neutrino number luminosity for the $15 M_{\odot}$ progenitor model. The origin of the horizontal axis corresponds to the time the dynamical simulation is started. Dotted and solid lines show the results for $\nu_{e}$ and $\bar{\nu}_{e}$, respectively. Colors distinguish the different reactions. In the collapse phase, only the total luminosity is shown for $\nu_{e}$ (pink dotted), since it is the quantity that the dynamical simulation provides. Note that the same number of $\nu_{e}$ and $\bar{\nu}_{e}$ is produced from electron-positron pair annihilations (red solid)

as shown in the legend. Note that for $\nu_{e}$ in the collapse phase we show only the total luminosity, since it is all that the transport calculations produce. The nuclear weak processes are considered in the NSE regions alone, and as a result, they arise only after the temperature reaches $T \gtrsim 5 \times 10^{9} \mathrm{~K}$. It is found that ECs on heavy nuclei and free protons are dominant in the emissions of $\nu_{e}$ during the progenitor phase, while the emissions of $\bar{\nu}_{e}$ occur mainly via electron-positron pair annihilation until around a few hundreds of seconds before collapse and thereafter $\beta^{-}$decay dominates, which is a new finding in this paper. Although $\nu_{e}$ overwhelms $\bar{\nu}_{e}$ in the collapse phase as expected, this is also true in the progenitor phase. It is particularly the case at $\sim 100 \mathrm{~s}$ prior to collapse when the ECs on free protons become appreciable.

Figure 6 is the same as Figure 5 but for the $12 M_{\odot}$ progenitor model. The results are similar to those of the $15 M_{\odot}$ model except that the numbers of emitted $\nu_{e}$ and $\bar{\nu}_{e}$ are slightly smaller for the $12 M_{\odot}$ model than for the $15 M_{\odot}$ model because the Fe core of the $12 M_{\odot}$ model has slightly higher densities and lower temperatures compared to the $15 M_{\odot}$ model (see Figure 3 ).

Figure 7 shows, on the other hand, the temporal evolutions of the number luminosities in the $9 M_{\odot}$ progenitor model, in which the ONe core collapses to produce an ECSN. The strong degeneracy of electrons suppresses electron-positron annihilation in this case, and as a result, the plasmon decay dominates initially until $60 \mathrm{~ms}$ after we switch to the hydrodynamical simulation when $\mathrm{Ne}$ and $\mathrm{O}$ are ignited at the center and the deflagration wave starts to propagate outward to produce NSE behind it. The yellow region corresponds to this $\mathrm{O}+\mathrm{Ne}$ deflagration phase in the figure. Then, $\bar{\nu}_{e}$ emissions by $\beta^{-}$decay and $\nu_{e}$ emissions via ECs on heavy nuclei as well as on free protons overtake those via plasmon decay.

In Figure 8, we present the radial profiles of the energy emissivities, $Q_{E}^{\nu}$, from different processes for the $15 M_{\odot}$ progenitor model at different times before collapse. The top panels display the results at a very early time in the progenitor phase $\left(\log _{10} \rho_{c} /\left[\mathrm{g} \mathrm{cm}^{-3}\right]=9.1\right)$, with both the radius (left) and mass coordinate (right) being employed as the horizontal axis. We define the $\mathrm{Fe}$ core as the region where the electron fraction

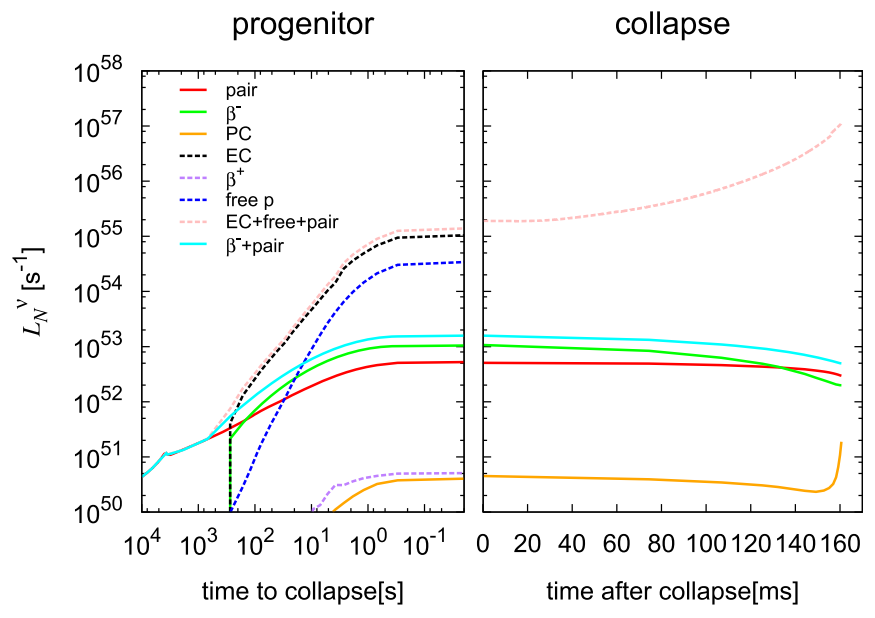

Figure 6. Same as Figure 5 but for the $12 M_{\odot}$ progenitor model.

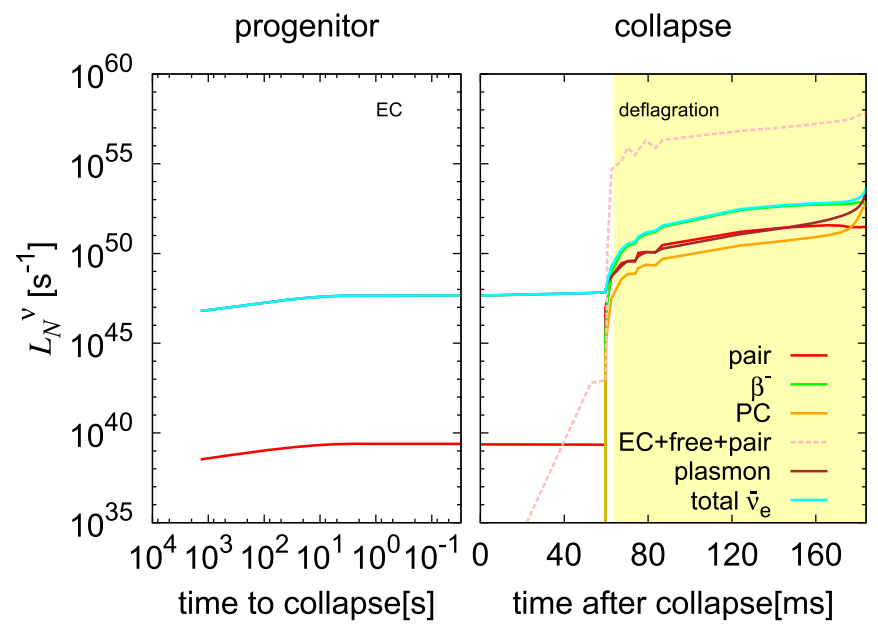

Figure 7. Same as Figures 5 and 6 but for the $9 M_{\odot}$ progenitor model. The yellow region corresponds to the phase in which the $\mathrm{O}+\mathrm{Ne}$ deflagration takes place.

satisfies $Y_{e}<0.495$, and show it in yellow. It is seen that all emissions occur rather uniformly in the region $r \lesssim 2 \times 10^{7} \mathrm{~cm}$ in this early phase. As the density increases with time, the $\bar{\nu}_{e}$ emissions are all suppressed toward the center, and the peaks in the emissivities appear off center and shifted to the peripheral, $r \sim 5 \times 10^{7} \mathrm{~cm}$, as shown in the bottom panels of the figure, which correspond to a later time $\left(\log _{10} \rho_{c} /\left[\mathrm{g} \mathrm{cm}^{-3}\right]=10.3\right)$. This is both due to the depletion of positrons in the initial state and to the Fermi-blocking of electrons in the final state as a consequence of the electron degeneracy. As for $\nu_{e}$ emissions, such a suppression does not occur, and the emissivities are greatest in the central region.

Figure 9 exhibits the differential luminosities or the energy spectra normalized by the corresponding total luminosities. The colors and types of lines are the same as those in Figure 5. One can see that the $\bar{\nu}_{e}$ emitted via PC on heavy nuclei (orange solid lines) have the highest average energies at all times. Recall, however, that the luminosity is very low for this process (see Figures 5-7). It should also be mentioned that the transport is not solved for $\bar{\nu}_{e}$, which will not be justified at high densities $\left(\log _{10} \rho_{c} /\left[\mathrm{g} \mathrm{cm}^{-3}\right] \gtrsim 11\right)$ for these high-energy $\bar{\nu}_{e}$. Regardless, the dominant process in the $\bar{\nu}_{e}$ emission is either electronpositron annihilation or $\beta^{-}$decay, and they both have average energies of $2-5 \mathrm{MeV}$ at most, which may justify ignoring the 


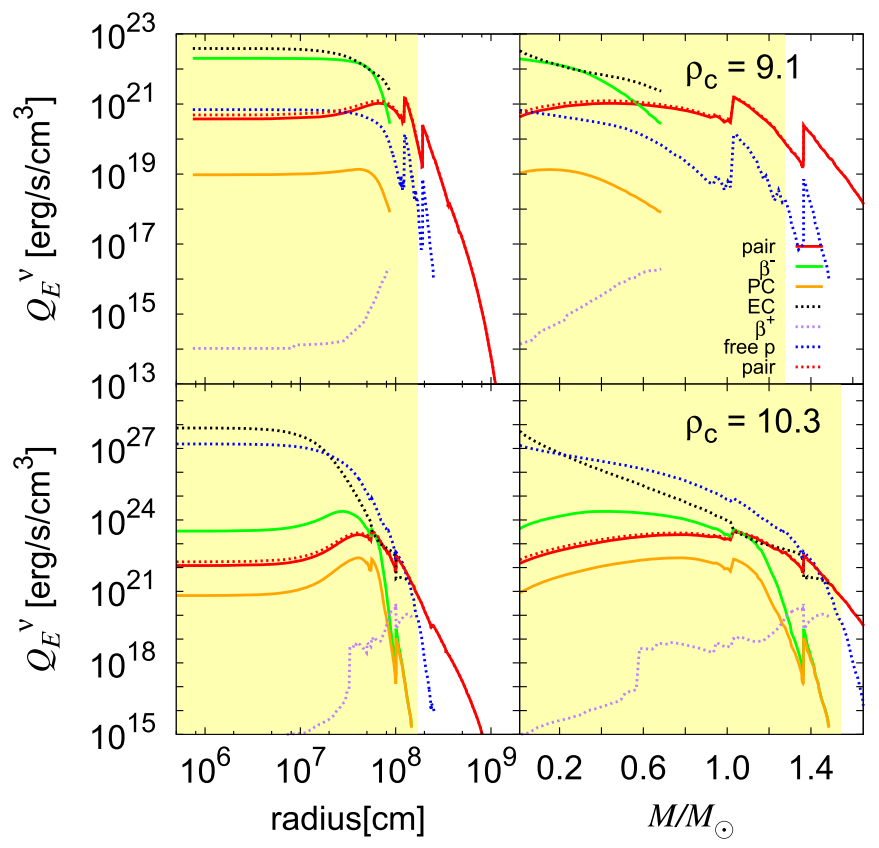

Figure 8. Radial profiles of the energy emissivities from different processes for the $15 M_{\odot}$ progenitor model. Top and bottom panels show the results when the central density is $\log _{10} \rho_{c} /\left[\mathrm{g} \mathrm{cm}^{-3}\right]=9.1$ and 10.3 , respectively. In the left panels, the radius is used as the horizontal axis, whereas in the right panels, the mass coordinate is employed. The line types and color coding are the same as in Figure 5. We define the Fe cores as the regions where the electron fraction satisfies $Y_{e}<0.495$, and they are in yellow in this figure.

transport. As for the $\nu_{e}$ emission, the ECs on heavy nuclei and free protons are mostly dominant and produce $\nu_{e}$ with $\sim 10 \mathrm{MeV}$. In this case, the transport in the core should be computed for a quantitative estimate of the luminosity and spectrum. A comparison between the results for the two types of progenitors indicates that neutrinos emitted from the ONe-core progenitor, especially those generated via electron-positron annihilation, have higher energies than those from Fe-core progenitors. This is because electrons are more strongly degenerate and have greater chemical potentials in the former.

In Table 2, we list the top five contributors to $\mathrm{EC}$ and $\beta^{-}$ decay, the dominant processes for producing $\nu_{e}$ and $\bar{\nu}_{e}$, respectively, at the time when $\log _{10} \rho_{c} /\left[\mathrm{g} \mathrm{cm}^{-3}\right]=10.3$ in the $15 M_{\odot}$ model. Note that EC occurs mostly in the central region, whereas $\beta^{-}$decay mainly happens off center. We hence evaluate the EC rates at $r=3.1 \times 10^{5} \mathrm{~cm}$, where the density, temperature, electron fraction, and chemical potential are $\log _{10} \rho /\left[\mathrm{g} \mathrm{cm}^{-3}\right]=10.3, T=0.861 \mathrm{MeV}, Y_{e}=0.417$, and $\mu_{e}=11.9 \mathrm{MeV}$. The $\beta^{-}$decay rates are presented, on the other hand, for the condition at $r=2.7 \times 10^{7} \mathrm{~cm}$, i.e., $\log _{10} \rho /\left[\mathrm{g} \mathrm{cm}^{-3}\right]=9.79, T=0.856 \mathrm{MeV}, Y_{e}=0.423$, and $\mu_{e}=7.87 \mathrm{MeV}$. We find that although the emissivities for individual nuclei are proportional to the product of their mass fraction and the reaction rate, the former is more important, since the latter changes by a factor whereas the former varies by an order. It is noteworthy in this respect that the top two contributors to EC and the top one to $\beta^{-}$decay are those nuclei with magic proton numbers, which is the reason why they are more abundant than others. Note again that their reaction rates are not the greatest.

In Figure 10, we show the energy spectra of neutrinos emitted from these nuclei. It is recognized that the spectra for the $\beta^{-}$decay presented in the lower panel are not much different among the nuclei. It is also evident that the average energies are lower than those for the $\nu_{e}$ emitted through ECs as exhibited in the upper panel. This is because the latter includes the contribution from the kinetic energy of degenerate electrons. The variation among the nuclei is also larger for EC.

Once NSE is established after the passage of the deflagration wave in the $9 M_{\odot}$ model, the composition is simply determined by the density, temperature, and electron fraction. The irongroup elements hence also become dominant for $\mathrm{EC}$ and $\beta^{-}$ decay in the $9 M_{\odot}$ model just as in the 12 and $15 M_{\odot}$ models.

\subsection{Event Numbers at Detectors}

Based on the results obtained so far, we estimate the numbers of detection events for some representative detectors, which include those under planning at present. For the detection of $\bar{\nu}_{e}$, almost all detectors utilize the inverse $\beta$ decay:

$$
\bar{\nu}_{e}+p \longrightarrow e^{+}+n \text {. }
$$

Following Odrzywolek et al. (2004), we express the crosssection $\sigma\left(E_{\nu}\right)$ of this interaction as

$$
\sigma\left(E_{\nu}\right)=0.0952\left(\frac{E_{e^{+} p_{e^{+}}}}{1 \mathrm{MeV}^{2}}\right) \times 10^{-42} \mathrm{~cm}^{2},
$$

in which the energy and three-momentum of the positron emitted are denoted by $E_{e^{+}}=E_{\nu}-\left(m_{n}-m_{p}\right)$ and $p_{e^{+}}=$ $\sqrt{E_{e^{+^{2}}}-m_{e}{ }^{2}}$, respectively.

Electron neutrinos are normally detected via electron scattering, $\nu_{e}+e^{-} \longrightarrow \nu_{e}+e^{-}$, in the currently available detectors. Its reaction rate is much lower than that of inverse $\beta$ decay, however, and the detection of $\nu_{e}$ in the pre-bounce phase has been thought to be almost impossible. Then, a new type of detector using liquid argon came into play. The planned deep underground neutrino observatory, or DUNE, is one such detector (DUNE collaboration 2016). It employs the absorption of $\nu_{e}$ by ${ }^{40} \mathrm{Ar}$,

$$
{ }^{40} \mathrm{Ar}+\nu_{e} \longrightarrow e^{-}+{ }^{40} \mathrm{~K}^{*} .
$$

The cross-section of this reaction is obtained numerically with SNOwGLoBES.

Then, the event rate, $r$, at a detector is expressed as

$$
r=\frac{N}{4 \pi D^{2}} \int_{E_{\mathrm{th}}}^{\infty} d E_{\nu_{1}} \sigma\left(E_{\nu_{1}}\right) \frac{d L_{N}^{\nu_{1}}}{d E_{\nu_{1}}}
$$

in which $N$ and $D$ denote the target number in the detector and the distance to the star from the detector, respectively. For simplicity, we assume that the detection efficiency is $100 \%$ above the threshold energy $E_{\mathrm{th}}$. The relevant features of the detectors that we consider in this paper, i.e., Super-Kamiokande, KamLAND, Hyper-Kamiokande, JUNO, and DUNE, are summarized in Table 3. The cumulative number of events, $N_{\text {cum }}$, is obtained by integrating the rate up to the given time,

$$
N_{\text {cum }}(t)=\int_{t_{\text {ini }}}^{t} r d t .
$$

In order to give quantitative estimates to the numbers of detection events, we need to appropriately take into account neutrino oscillations. For this purpose, it is not only the luminosities of electron-type neutrinos but also those of $\mu$ - and $\tau$-type neutrinos that are required. In this paper, we calculated them for electron-positron annihilation in the same way as $\nu_{e}$ 

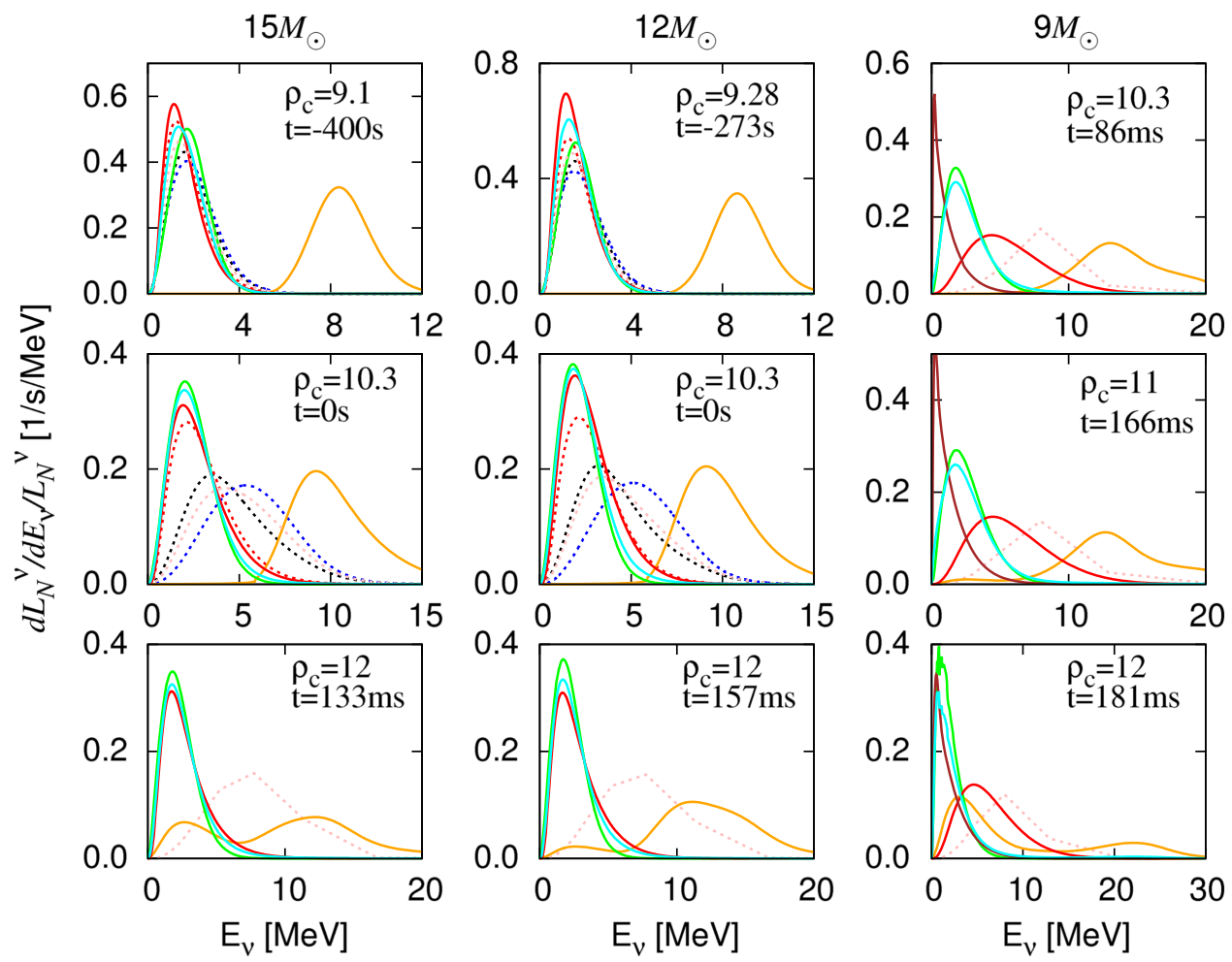

Figure 9. Spectra of neutrinos emitted from the entire star at indicated times for the three progenitor models. They are normalized by the corresponding number luminosities. Colors indicate different emission processes as in Figure 5. Note that the scale of the horizontal axes is different among the three models.

and $\bar{\nu}_{e}$. We give the results in Figure 11, in which the time evolution of the number luminosities as well as the energy spectra at three different epochs are displayed in the upper and lower panels, respectively. It is observed that their luminosities are much lower than those of $\nu_{e}$ as expected and are somewhat lower even compared with $\bar{\nu}_{e}$. This is simply because $\mu$ - and $\tau$-type neutrinos lack charged-current reactions and are produced solely from electron-positron annihilation. The average energies are $\lesssim 2 \mathrm{MeV}$, much lower than those of $\nu_{e}$ and as a result, the opacities for these heavy-lepton neutrinos are smaller, justifying the neglect of transport in their calculations.

Figures 12-14 present the time evolutions of the event rates (top) and cumulative numbers of detection events (bottom) for different detectors in the progenitor (left) and collapse (right) phases for the three progenitors. For the $9 M_{\odot}$ model, only the collapse phase is shown, since the progenitor phase will not be observed even at a distance as close as 200 pc (Paper I). The normal (inverted) hierarchy is assumed in the upper (lower) half of the top panels in each figure. All of the detectors except DUNE, which will detect $\nu_{e}$, will mainly observe $\bar{\nu}_{e}$. From a comparison of the left and right panels, we find that the progenitor phase is dominant over the collapse phase for $\bar{\nu}_{e}$, with the latter contributing only a few percent. This is due to the electron degeneracy, which suppresses both $\beta^{-}$decay via Fermi-blocking of the electron in the final state and electronpositron annihilation through the depletion of the positron in the initial state.

In the case of $\nu_{e}$, the collapse phase is much more important although it lasts for much shorter periods. This is because both the luminosity and average energy increase with density. The detections of $\bar{\nu}_{e}$ in the pre-bounce phase are hence more suitable as an alert of an imminent supernova (Asakura et al. 2016; Yoshida et al. 2016). In fact, we may be able to issue an alert a few days before core collapse for Fe-core progenitors if neutrinos obey the normal mass hierarchy. The $\nu_{e}$ emissions from the ONe-core progenitor, on the other hand, are much shorter than those from the $\mathrm{Fe}$-core progenitors presented in Figure 14. They become appreciable only after NSE is established in the collapsing core by the passage of the deflagration wave. DUNE will only detect $\nu_{e}$ less than $100 \mathrm{~ms}$ prior to bounce, and may hence make it possible to distinguish between the two types of progenitors by the time of the first detection of $\nu_{e}$.

Depending on the mass hierarchy, the neutrino oscillations predominantly affect either $\nu_{e}$ or $\bar{\nu}_{e}$. In fact, in the normal hierarchy, the spectrum of $\nu_{e}$ is exchanged with that of $\nu_{\tau}$ in the adiabatic MSW oscillation and is further mixed among three flavors in the vacuum oscillations, whereas the spectrum of $\bar{\nu}_{e}$ is mixed with those of $\bar{\nu}_{\mu}$ and $\bar{\nu}_{\tau}$ only in the vacuum oscillations. The situation is the other way around in the case of the inverted hierarchy, in which MSW also affects $\bar{\nu}_{e}$. Recall that the luminosities of $\nu_{x}$ and $\bar{\nu}_{x}$ are lower than those of $\nu_{e}$ and $\bar{\nu}_{e}$.

As a consequence, the chance to observe $\bar{\nu}_{e}$ is higher for the normal hierarchy, and JUNO will see more than 850 of them in the progenitor phase from as early as a few days prior to collapse, which is roughly the end of $\mathrm{O}$ burning, if the $15 M_{\odot}$ progenitor is located $200 \mathrm{pc}$ from the Earth. The event number will be reduced by a factor of $\sim 4$ in the case of inverted hierarchy. The detection of $\nu_{e}$ by DUNE will be more plausible for the inverted hierarchy and, in fact, the expected event number may exceed 2000 if the distance to the source is again 200 pc, i.e., the distance to Betelgeuse and the energy threshold is optimistically assumed to be $5 \mathrm{MeV}$. The first $\nu_{e}$ may be observed several tens of minutes before collapse, which corresponds to the end of Si burning. In the normal hierarchy, on one hand, the number of detections will be reduced by more 
Table 2

Weak Reaction Rates and Mass Fractions of the Top Five Nuclei Contributing to the Total Number Luminosities from EC and $\beta^{-}$Decay in the $15 M_{\odot}$ Progenitor Model at the Time When the Central Density is $\log _{10} \rho_{c} /\left[\mathrm{g} \mathrm{cm}^{-3}\right]=10.3^{\mathrm{a}}$

\begin{tabular}{|c|c|c|c|c|c|c|c|}
\hline \multicolumn{4}{|c|}{$\mathrm{EC}^{\mathrm{b}}$} & \multicolumn{4}{|c|}{$\beta^{-\mathrm{c}}$} \\
\hline & $(N, Z)$ & $X_{i}$ & $R_{i}$ & & $(N, Z)$ & $X_{i}$ & $R_{i}$ \\
\hline$\overline{{ }^{66} \mathrm{Ni}}$ & $(38,28)$ & $7.76 \times 10^{-2}$ & 10.57 & ${ }^{49} \mathrm{Ca}$ & $(29,20)$ & $1.88 \times 10^{-2}$ & $3.64 \times 10^{-2}$ \\
\hline${ }^{76} \mathrm{Ge}$ & $(44,32)$ & $5.88 \times 10^{-3}$ & 32.59 & ${ }^{65} \mathrm{Co}$ & $(38,27)$ & $4.60 \times 10^{-3}$ & $1.78 \times 10^{-1}$ \\
\hline${ }^{87} \mathrm{Kr}$ & $(51,36)$ & $7.85 \times 10^{-3}$ & 26.37 & ${ }^{59} \mathrm{Mn}$ & $(34,25)$ & $9.78 \times 10^{-3}$ & $5.20 \times 10^{-2}$ \\
\hline${ }^{70} \mathrm{Zn}$ & $(40,30)$ & $5.32 \times 10^{-3}$ & 30.04 & ${ }^{55} \mathrm{~V}$ & $(32,23)$ & $6.05 \times 10^{-3}$ & $7.62 \times 10^{-2}$ \\
\hline
\end{tabular}

Notes.

a This density corresponds to the time when we switch to the dynamical calculation $(t=0)$.

${ }^{\mathrm{b}}$ The EC rates are evaluated at $r=3.1 \times 10^{5} \mathrm{~cm}$, where the density, temperature, electron fraction, and chemical potential are $\log _{10} \rho /\left[\mathrm{g} \mathrm{cm}^{-3}\right]=10.3$, $T=0.861 \mathrm{MeV}, Y_{e}=0.417$, and $\mu_{e}=11.9 \mathrm{MeV}$.

${ }^{\mathrm{c}}$ The rates of $\beta^{-}$decays are calculated at $r=2.7 \times 10^{7} \mathrm{~cm}$, where they are largest and $\log _{10} \rho /\left[\mathrm{g} \mathrm{cm}^{-3}\right]=9.79, T=0.856 \mathrm{MeV}, Y_{e}=0.423$, and $\mu_{e}=7.87 \mathrm{MeV}$.

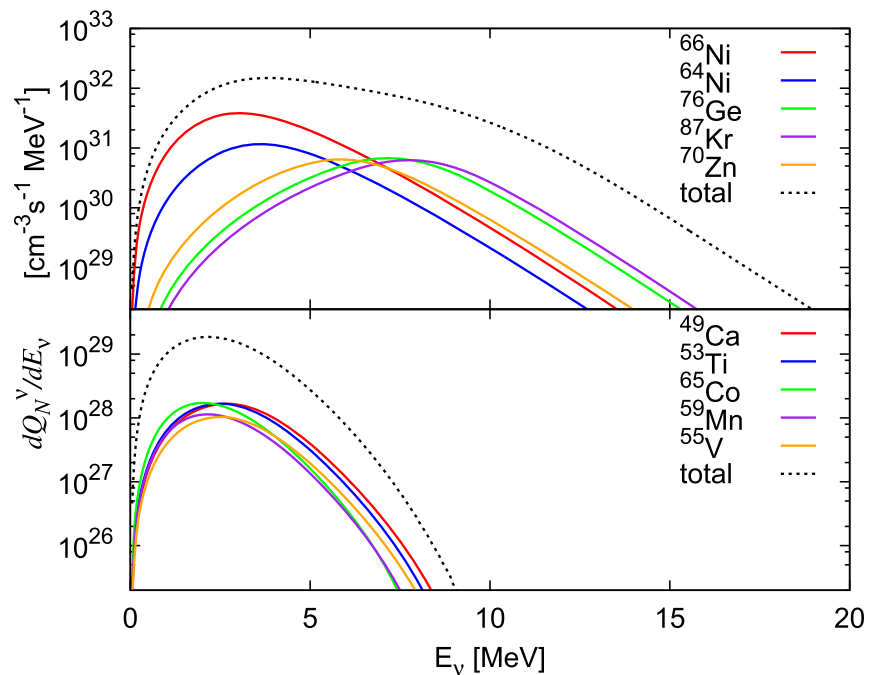

Figure 10. Energy spectra for EC and $\beta^{-}$decay by the dominant heavy nuclei given in Table 2 at the time when the central density becomes $\log _{10} \rho_{c} /\left[\mathrm{g} \mathrm{cm}^{-3}\right]=10.3$. The top panel shows the $\nu_{e}$ spectrum for EC, while the bottom panel displays the $\bar{\nu}_{e}$ spectrum for $\beta^{-}$decay. The EC rates are evaluated at $r=3.1 \times 10^{5} \mathrm{~cm}$, where the density, temperature, electron fraction, and chemical potential $\operatorname{are} \log _{10} \rho /\left[\mathrm{g} \mathrm{cm}^{-3}\right]=10.3, T=0.861 \mathrm{MeV}$, $Y_{e}=0.417$, and $\mu_{e}=11.9 \mathrm{MeV}$, respectively. The rates of $\beta^{-}$decay are calculated at $r=2.7 \times 10^{7} \mathrm{~cm}$, where they are largest, and $\log _{10} \rho /\left[\mathrm{g} \mathrm{cm}^{-3}\right]=$ $9.79, T=0.856 \mathrm{MeV}, Y_{e}=0.423$, and $\mu_{e}=7.87 \mathrm{MeV}$.

than a factor of 10 . Such a large difference in the number of detections suggests a possibility of judging the neutrino mass hierarchy. It is interesting to point out that as far as $\nu_{e}$ is concerned, the ONe-core progenitor may offer a better chance of detection at DUNE. This is because the temperature in the NSE region behind the $\mathrm{O}+\mathrm{Ne}$ deflagration is higher than that in the Fe core. As far as the Fe-core progenitors are concerned, the more massive it is, the larger the number of detection events expected for both $\nu_{e}$ and $\bar{\nu}_{e}$.

In Table 4, we summarize the expected numbers of events at Super-Kamiokande, KamLAND, Hyper-Kamiokande, JUNO, and DUNE, assuming that the progenitors are $200 \mathrm{pc}$ away from us. These are the numbers of $\nu_{e}$ events for DUNE, and the numbers of $\bar{\nu}_{e}$ events for other detectors. In the table, the contributions from both the progenitor and collapse phases are exhibited. It is found that $\bar{\nu}_{e}$ from the 12 and $15 M_{\odot}$ progenitors can be detected at all detectors if the source is this close. In particular, the planned detectors such as Hyper-Kamiokande
Table 3

The Detector Parameters Assumed in this Paper ${ }^{\mathrm{a}, \mathrm{b}, \mathrm{c}}$

\begin{tabular}{lrcc}
\hline \hline Detector & $\begin{array}{c}\text { Mass } \\
(\mathrm{kt})\end{array}$ & $\begin{array}{c}\text { Target Number } \\
N\end{array}$ & $\begin{array}{c}\text { Energy Threshold } \\
(\mathrm{MeV})\end{array}$ \\
\hline Super-K & 32 & $2.14 \times 10^{33}$ & 5.3 \\
KamLAND & 1 & $8.47 \times 10^{31}$ & 1.8 \\
Hyper-K & 516 & $3.45 \times 10^{34}$ & 8.3 \\
JUNO & 20 & $1.69 \times 10^{33}$ & 1.8 \\
DUNE & 40 & $6.02 \times 10^{32}$ & $5.0,10.8$ \\
\hline
\end{tabular}

Notes.

${ }^{\mathrm{a}}$ The numbers given here are not very precise and are just meant as a rough estimate. JUNO is assumed to be a scale up of KamLAND by a factor of 20 . We also assume that the energy threshold of Hyper-Kamiokande will be somewhat higher than that of Super-Kamiokande.

${ }^{\mathrm{b}}$ We use the total volume for the two-tank design of Hyper-Kamiokande.

${ }^{c}$ The energy threshold of DUNE is still uncertain, and we employ both optimistic $(5 \mathrm{MeV})$ and more realistic $(10.8 \mathrm{MeV})$ values in this study.

References. (1) Super-Kamiokande collaboration (2014), (2) KamLAND collaboration (2009), (3) Abe (2016), (4) An (2016), (5) DUNE collaboration (2016).

and JUNO look promising if one considers the number of events alone: they will detect a few tens of $\bar{\nu}_{e}$ even if they are emitted $1 \mathrm{kpc}$ away. The detection of $\bar{\nu}_{e}$ from ONe-core progenitor seems to be nearly impossible even with the planned detectors. We will hence be able to distinguish the two types of progenitors, i.e., ONe-core progenitors and $\mathrm{Fe}$-core progenitors, by the detection or non-detection of $\bar{\nu}_{e}$, the same conclusion as in Paper I. We stress, however, that in this paper, we incorporated nuclear processes, such as $\beta^{-}$decay, which were ignored in Paper I but are demonstrated to dominate in the production of $\bar{\nu}_{e}$. We show the expected numbers of $\nu_{e}$ events for the values of two energy thresholds, 5 and $10.8 \mathrm{MeV}$, considering its uncertainties at present. The former is somewhat optimistic and the latter may be more realistic. The first detection of $\nu_{e}$ will be delayed for the latter case to a few tens of seconds before core bounce. Note, however, that the energy of $\nu_{e}$ in the collapse phase is high $(\sim 8 \mathrm{MeV})$, and we will be still able to detect a large number of $\nu_{e}$. In this paper, we do not treat the neutrino emissions at $\log _{10} \rho /\left[\mathrm{g} \mathrm{cm}^{-3}\right]>13$ because the compositions and weak reaction rates of heavy nuclei are highly uncertain there. The number of events for $\bar{\nu}_{e}$ will not increase much by the time of core bounce, however. In fact, it is expected to increase by 

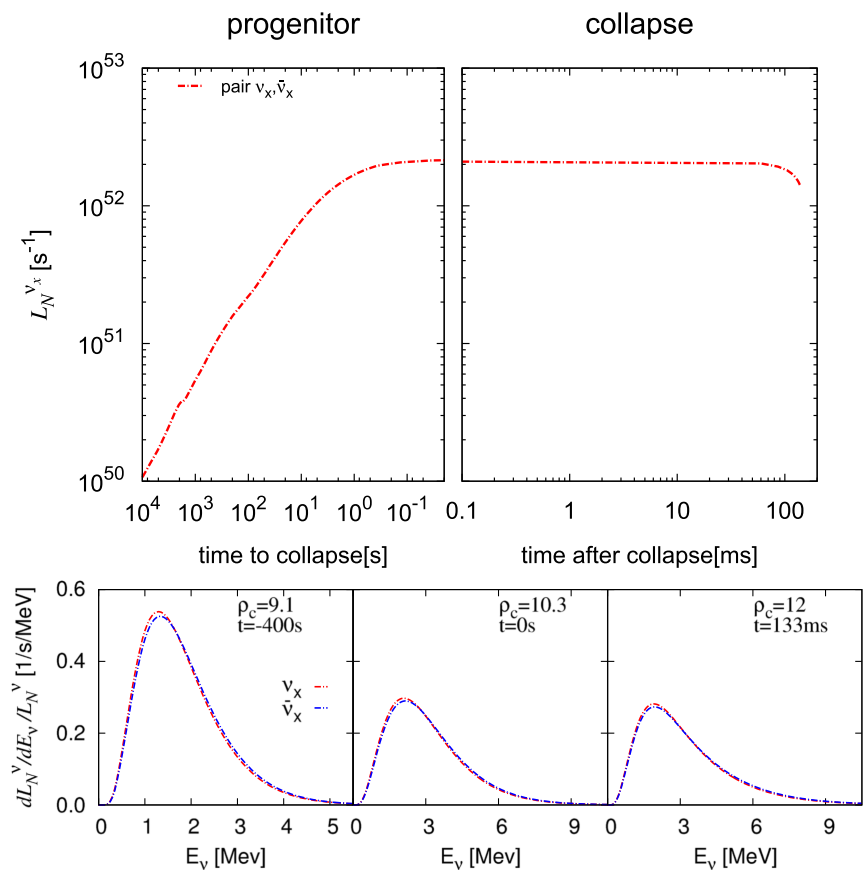

Figure 11. Number luminosity (top) and normalized spectra (bottom) of $\nu_{x}$ and $\bar{\nu}_{x}$ emitted via electron-positron annihilation. Note that the number luminosities of $\nu_{x}$ and $\bar{\nu}_{x}$ are identical to each other.

$\sim 200$ for $\nu_{e}$ if one simply extrapolates the results obtained for $\log _{10} \rho_{c} /\left[\mathrm{g} \mathrm{cm}^{-3}\right]<13$ up to core bounce in the inverted hierarchy. This issue will be addressed in a future publication.

\section{Summary and Discussions}

In our previous paper (Paper I), we calculated $\bar{\nu}_{e}$ emissions via thermal processes alone: the electron-positron pair annihilation and plasmon decay from both the Fe-core and ONe-core progenitors. The nuclear weak processes, i.e., the $\beta^{\mp}$ decays, and electron and positron captures, were ignored, however. Moreover, neutrino emissions in the collapse phase were not considered either, because computations of the hydrodynamics and neutrino transfer would have been required. Ignoring these is no longer justified as liquid $\mathrm{Ar}$ detectors such as DUNE have come into view to detect $\nu_{e}$, which are predominant in the collapse phase but are difficult to observe with the existing detectors. It should be stressed here that no quantitative estimate has been done so far on the neutrino emissions during the collapse phase mainly because neutrinos are emitted more intensively in the post-bounce phase and the protoneutron star cooling phase that follows. This paper is hence the first to demonstrate that the collapsing phase has the potential to provide new insights.

In this paper, we investigated the emissions of all types of neutrinos from the progenitor phase up to the pre-bounce time, when the central density reaches $\log _{10} \rho_{c} /\left[\mathrm{g} \mathrm{cm}^{-3}\right]=13$. We compared the two types of progenitors of CCSNe: one that produces an $\mathrm{Fe}$ core and the other that yields an $\mathrm{ONe}$ core before core collapse, to see whether we can get some information on the cores deep inside massive stars, which would be inaccessible by other means, by observing the neutrinos they emit. We first recalculated the neutrino emissions from realistic progenitor models with 9,12 , and $15 M_{\odot}$ on the zero age main sequence with both thermal and nuclear weak processes taken into account. Note again that the $9 M_{\odot}$ model is
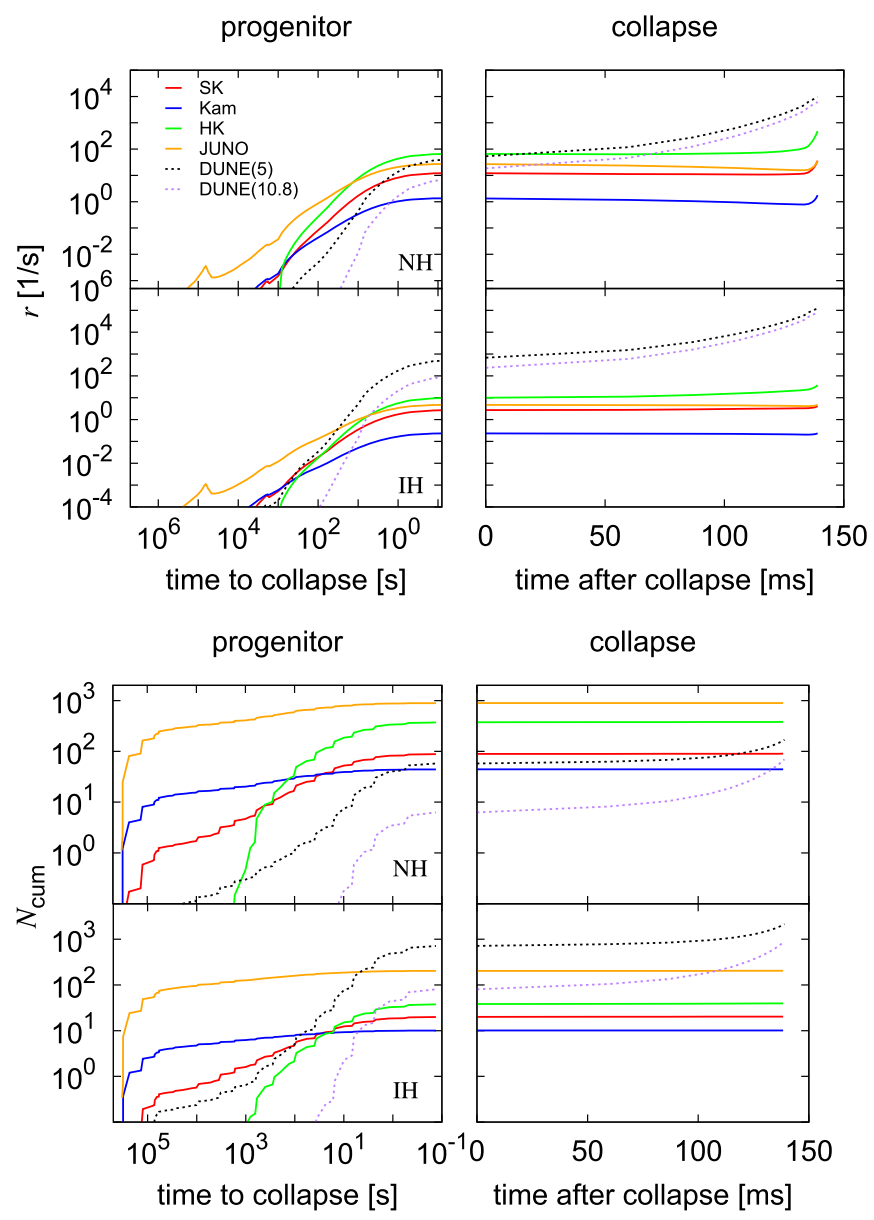

Figure 12. Time evolutions of the event rates (top panels) and cumulative numbers of events (bottom panels) for the $15 M_{\odot}$ progenitor model. The upper half of each panel shows the results for the normal mass hierarchy, while the lower half presents those for the inverted mass hierarchy. Colors specify the neutrino detectors. We consider $\nu_{e}$ for DUNE (dotted line) and $\bar{\nu}_{e}$ for other detectors (solid lines).

a progenitor with an $\mathrm{ONe}$ core that collapses to produce an ECSN, and the other two are supposed to be progenitors of FeCCSNe.

We then switched to hydrodynamical simulations of core collapse up to the pre-bounce time, when the central density reaches $\log _{10} \rho_{c} /\left[\mathrm{g} / \mathrm{cm}^{3}\right]=13$, with the transfer of $\nu_{e}$ in the core being treated appropriately. Since other types of neutrinos are much less abundant and have lower energies typically, we treated them in the post-process, in which we extracted the time evolutions of the density, temperature, $Y_{e}$, and $f_{\nu_{e}}$, the distribution function of $\nu_{e}$, from the results of the simulations and calculated the emissivities of these neutrinos with the possible minor back-reactions to dynamics being ignored. Finally, based on the luminosities and spectra of neutrinos thus obtained, we estimated the expected numbers of detection events on some representative neutrino detectors. In so doing, we took into proper account the vacuum and MSW oscillations of neutrino flavors.

We found that the $\beta^{-}$decay and the EC on heavy nuclei and free protons dominate the number luminosities of $\bar{\nu}_{e}$ and $\nu_{e}$, respectively, from several tens of minutes before core bounce. Heavy nuclei, not with large reaction rates but with large mass fractions, contribute the most to these reactions. Because of the Fermi-blocking of electrons in the final state, $\beta^{-}$decay is 
Table 4

Expected Numbers of Detection Events for Different Detectors ${ }^{\mathrm{a}, \mathrm{b}}$

\begin{tabular}{|c|c|c|c|c|c|c|}
\hline \multirow[t]{2}{*}{ Detector } & \multicolumn{2}{|c|}{$9 M_{\odot}$} & \multicolumn{2}{|c|}{$12 M_{\odot}$} & \multicolumn{2}{|c|}{$15 M_{\odot}$} \\
\hline & Normal & Inverted & Normal & Inverted & Normal & Inverted \\
\hline Super-K & 0.93 & 0.03 & $\begin{array}{c}30.8 \\
(30.1+0.71)\end{array}$ & $\begin{array}{c}8.68 \\
(8.48+0.20)\end{array}$ & $\begin{array}{c}89.9 \\
(88.3+1.61)\end{array}$ & $\begin{array}{c}20.3 \\
(19.9+0.41)\end{array}$ \\
\hline KamLAND & 0.05 & 0.002 & $\begin{array}{c}32.0 \\
(31.9+0.07)\end{array}$ & $\begin{array}{c}9.15 \\
(9.13+0.02)\end{array}$ & $\begin{array}{c}44.3 \\
(44.2+0.15)\end{array}$ & $\begin{array}{c}10.1 \\
(10.1+0.03)\end{array}$ \\
\hline Hyper-K & 11.6 & 0.42 & $\begin{array}{c}83.9 \\
(80.0+3.85)\end{array}$ & $\begin{array}{c}10.9 \\
(10.1+0.76)\end{array}$ & $\begin{array}{c}363 \\
(353+9.84)\end{array}$ & $\begin{array}{c}37.7 \\
(35.9+1.82)\end{array}$ \\
\hline DUNE (5 MeV) & 1765 & 22685 & $\begin{array}{c}137 \\
(32.4+105)\end{array}$ & $\begin{array}{c}1756 \\
(406+1350)\end{array}$ & $\begin{array}{c}169 \\
(57.8+111)\end{array}$ & $\begin{array}{c}2142 \\
(713+1429)\end{array}$ \\
\hline DUNE (10.8 MeV) & 1238 & 15910 & $\begin{array}{c}61.3 \\
(3.33+58.0)\end{array}$ & $\begin{array}{c}789 \\
(42.7+746)\end{array}$ & $\begin{array}{c}69.3 \\
(6.27+63)\end{array}$ & $\begin{array}{c}895 \\
(80.1+815)\end{array}$ \\
\hline
\end{tabular}

Notes.

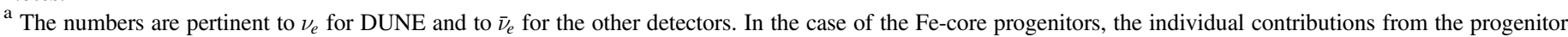
and collapse phases are also shown in parentheses in this order.

${ }^{\mathrm{b}}$ The source is assumed to be located $200 \mathrm{pc}$ from the Earth. Both normal and inverted mass hierarchies are considered in the adiabatic oscillation limit.

suppressed at high densities, where electrons are strongly degenerate, and the number luminosity of $\bar{\nu}_{e}$ is decreased toward core bounce. As a consequence, the progenitor phase is dominant over the collapse phase in $\bar{\nu}_{e}$ emission. In contrast, $\nu_{e}$ emission occurs predominantly in the collapse phase, although it is much shorter than the progenitor phase that precedes it. The detection of $\bar{\nu}_{e}$ in the pre-SN phase is hence more suitable as an alert of an imminent supernova, which may indeed be possible a few days before core bounce for Fe-core progenitors if neutrinos obey the normal mass hierarchy.

The $\bar{\nu}_{e}$ from the 12 and $15 M_{\odot}$ progenitors can be detected by all detectors, especially on the planned detectors such as Hyper-Kamiokande and JUNO if the distance to them is $\lesssim 1 \mathrm{kpc}$. The $\bar{\nu}_{e}$ from the $9 M_{\odot}$ progenitor will be quite difficult to observe even if it is as close to us as $200 \mathrm{pc}$, the distance to Betelgeuse. We may hence conclude that we can distinguish the two types of progenitors by the detection or non-detection of $\bar{\nu}_{e}$ prior to collapse. With DUNE, on the other hand, we will be able to detect more than a thousand $\nu_{e}$ from all the progenitor models if the distance to the source is again $200 \mathrm{pc}$ and neutrinos follow the inverted mass hierarchy. The event numbers are reduced by a factor of $\sim 10$ if they obey the normal mass hierarchy. Such a large difference in the number of detections suggests a possibility of judging the neutrino mass hierarchy. It is interesting to see that the ONe-core progenitor offers the best chance in this case. This implies that regardless of the type and mass of the progenitor, we may be able to confirm our current understanding of the physics in the collapse phase. Note, however, that $\nu_{e}$ are not useless in distinguishing progenitor types. Although it will not be easy observationally, the fact that $\nu_{e}$ emissions from the $\mathrm{ONe}$-core progenitor in the pre-bounce phase occur in much shorter periods than those from the Fe-core progenitors may be utilized to discriminate the former from the latter.

Our estimates admittedly include several uncertainties. In the following, we comment on them in turn. In this paper, we began the hydrodynamical simulations of the collapse phase when the central density becomes $\log _{10} \rho_{c} /\left[\mathrm{g} \mathrm{cm}^{-3}\right]=10.3$ for the Fe-core progenitors, which is rather arbitrary. In fact, the cores are already unstable at this point and have started to collapse in the quasi-static evolutionary calculations, which means that we could have switched to the dynamical simulations a bit earlier. Indeed, if we switch at $\log _{10} \rho_{c} /\left[\mathrm{g} \mathrm{cm}^{-3}\right]=10$, the time it takes to reach core bounce is shortened by more than a second. This is due to artificially accelerated collapse in the new calculation, which is in turn caused mainly by differences between the EOS used in the stellar evolution calculation and that employed in the dynamical simulation. The EC rates are also different. Although the discrepancy of more than a second in the time up to core bounce may not seem small, the difference in the event numbers might not be that large, since most of the deviation occurs immediately after the onset of the simulation, when the density is still not very high.

The uncertainty in the EOS also affects the EC rates through the mass fractions of heavy nuclei in the NSE composition. Buyukcizmeci et al. (2013) compared the nuclear composition of three multispecies EOSs, including ours, that were recently constructed for supernova simulations. According to their results, differences in the mass fractions of heavy nuclei increase with temperature and/or density and become as large as a factor of two at $T=2 \mathrm{MeV}$ and $\log _{10} \rho /\left[\mathrm{g} \mathrm{cm}^{-3}\right]=11$. The different treatments of the surface, bulk, and shell energies of heavy nuclei are the main causes for the discrepancies. In fact, the temperature dependence of the shell energies that is incorporated in Furusawa et al. (2017) tends to smooth out the mass distribution around closed shell nuclei and may reduce the EC rate at early times in the collapse phase by $\sim 20 \%$ (Furusawa et al. 2017). The shell quenching considered in Raduta et al. (2016) may also affect the nuclear weak rates during the collapse phase.

As explained in Section 3, we employed the nuclear weak interaction rates obtained by detailed calculations for individual heavy nuclei whenever they are available. As the density and temperature increase in the collapse phase, however, heavy nuclei that are not included in these tables appear. We are then forced to use for these nuclei the approximation formula, Equation (22), for EC and another table (Tachibana \& Yamada 1995) for $\beta^{-}$decay. Since the approximation formula is based on the data of nuclei around the $\beta$ stable line, it may not be applicable to neutron-rich nuclei. The rates in Tachibana's table, on the other hand, were not meant for 

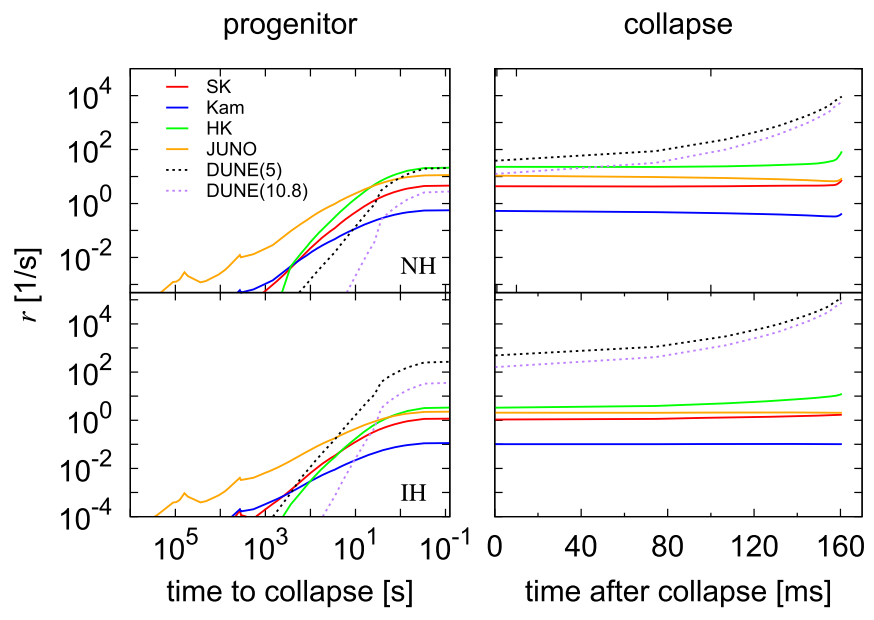

progenitor
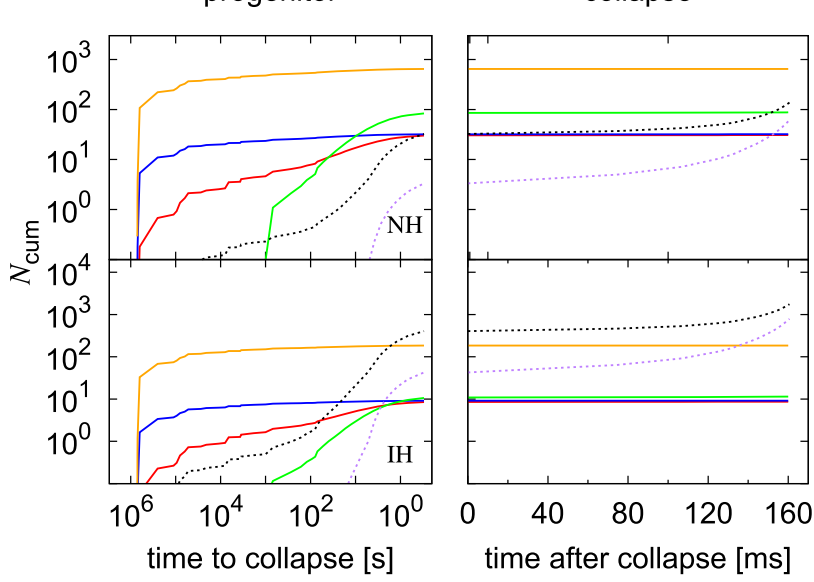

Figure 13. Same as Figure 12 but for the $12 M_{\odot}$ progenitor.

supernova simulations originally and were calculated for isolated nuclei under the terrestrial condition. We hence included the Fermi-blocking of electrons in the final state very crudely. Moreover, the data in this table do not include the contribution from excited states. When the central density exceeds $\log _{10} \rho_{c} /\left[\mathrm{g} \mathrm{cm}^{-3}\right] \sim 11.4$, most $\bar{\nu}_{e}$ come from the $\beta^{-}$ decays of nuclei, the rates of which are derived from this table. We certainly need to improve them in the future. In this paper, we have not treated the neutrino emissions at $\log _{10} \rho /\left[\mathrm{g} \mathrm{cm}^{-3}\right] \gtrsim 13$ on purpose because nuclei become more and more exotic with their mass and atomic numbers getting larger, producing so-called nuclear pastas before uniform nuclear matter is realized. The compositions and weak reaction rates of these nuclei are highly uncertain at such high densities. Moreover, dynamical simulations handle them in a very crude way, ignoring a possible variety of pasta configurations and interpolating the reaction rates between a certain subnuclear density and the nuclear saturation density. As mentioned earlier, one can crudely estimate the number of detections of $\nu_{e}$ during the period from $\log _{10} \rho_{c} /\left[\mathrm{g} \mathrm{cm}^{-3}\right]=13$ until core bounce by simply extrapolating the event rates obtained in Figures 12-14. We found then that $\sim 200$ more $\nu_{e}$ may be observed by DUNE for the inverted mass hierarchy. We certainly need improvements in the treatment of this phase, which will be future work.

Although it is much beyond the scope of this paper to take into account in detail the background noise for each detector and discuss the detection possibility quantitatively, we touch
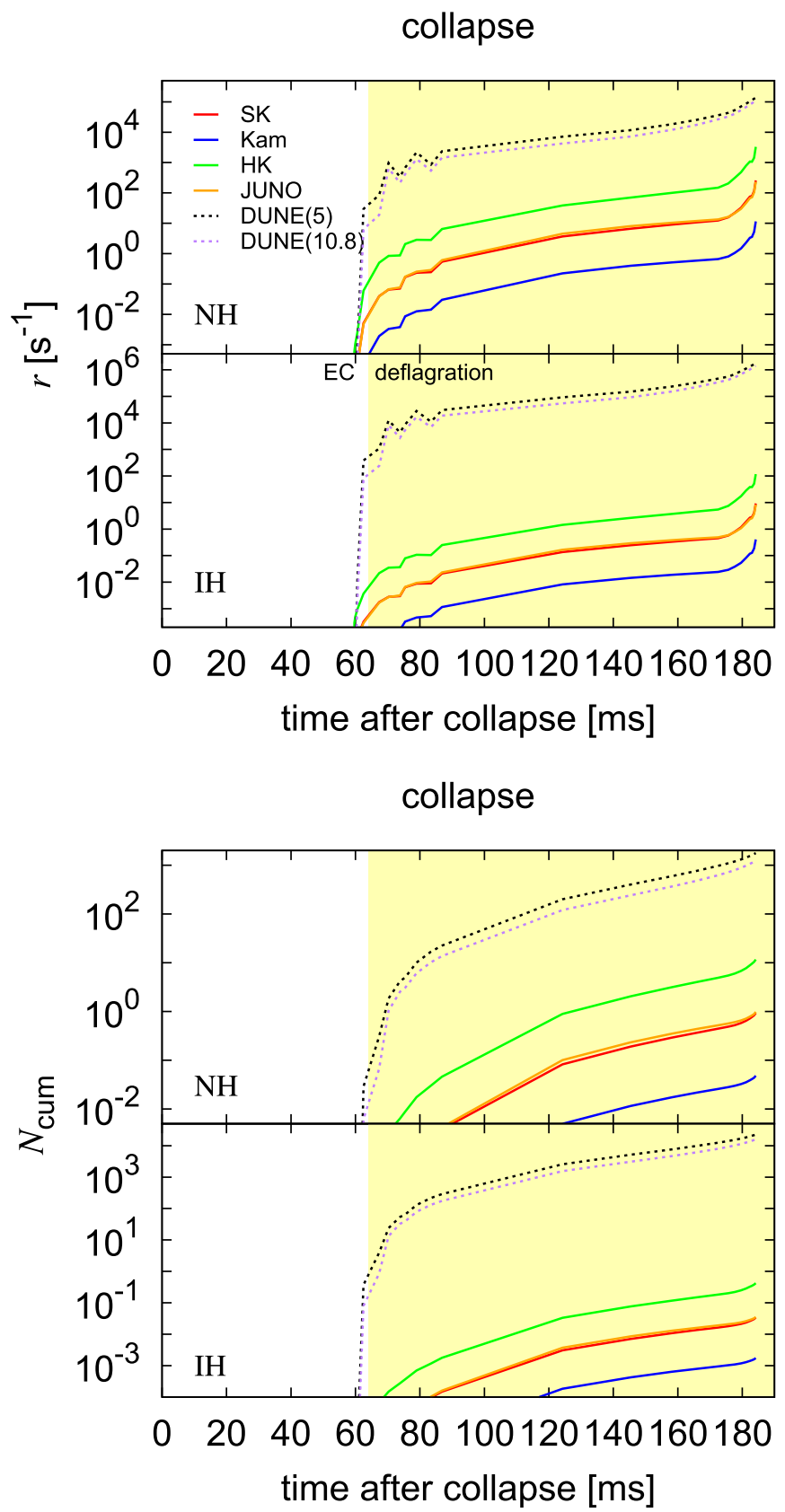

Figure 14. Same as Figures 12 and 13 but for the $9 M_{\odot}$ progenitor. Only the collapse phase is shown.

on this issue briefly, since the actual detectability crucially depends on it. If we adopt several hundreds of events/day as the typical noise level of Super-Kamiokande at present, $\bar{\nu}_{e}$ may not be detected even from FeCCSNe located at 200 pc. However, the background will be reduced remarkably to 0.21 events per hour after gadolinium is doped as designed (Beacom $\&$ Vagins 2004). An accompanied reduction of the energy threshold may increase the number of events by a factor of $\gtrsim 10$ as demonstrated by Yoshida et al. (2016). The background for KamLAND is already very low, $\sim 1$ event/day, and will not be a problem. In the case of Hyper-Kamiokande, on the other hand, the reduction of the energy threshold, if possible, will have a big impact on the event number as mentioned earlier.

In this paper, we considered only two relatively light $\mathrm{Fe}$-core progenitors. It is certainly important, though, to study other 
more massive progenitors systematically. It should also be emphasized that the expected event numbers for the present models may change by a factor of a few if one considers various uncertainties in the current stellar evolution calculation. As stated at the beginning, our ultimate goal is to extend seamlessly and consistently the current investigation until the end of the cooling phase of proto-neutron stars. We stress again that most of the studies on neutrino emissions from CCSNe and their detections at terrestrial detectors done so far have treated the post-core bounce phase and the subsequent phase of the protoneutron star cooling separately, and very little attention has been paid to the phase preceding them. Now that we have a lot of CCSN simulations that are successful in obtaining explosions, we believe that we should make a serious effort to draw the light curves and spectral evolutions of neutrinos that span the entire period from the progenitor phase up to the formation of the normal neutron star. This paper is just the first step.

We are grateful to Dr. Tachibana for providing us with the table of nuclear weak interaction rates and to Dr. Beacom for his useful advice. This work is partly supported by Grants-in-Aid for Scientific Research from the Ministry of Education, Culture, Sports, Science and Technology of Japan (Nos. 24244036, 24103006, 26104007, 26400220, 26400271), and the HPCI Strategic Program of Japanese MEXT. H.N. and S.F. are supported by the Japan Society for the Promotion of Science Postdoctoral Fellowships for Research Abroad. Some numerical calculations were carried out on the PC cluster at the Center for Computational Astrophysics, National Astronomical Observatory of Japan. K.T. is supported by Overseas Research Fellowships of the Japan Society for the Promotion of Science (JSPS).

\section{ORCID iDs}

Chinami Kato (D) https://orcid.org/0000-0003-4213-0076

Hiroki Nagakura (iD https://orcid.org/0000-0002-7205-6367

Koji Ishidoshiro (i) https://orcid.org/0000-0001-9271-2301

Shoichi Yamada (iD https://orcid.org/0000-0002-2166-5605

\section{References}

Abe, K., Abe, Ke., \& Aihara, H. 2016, KEK preprint 2016-21 (https://libextopc.kek.jp/preprints/PDF/2016/1627/1627021.pdf)

An, F. 2016, JPhG, 43, 3

Arnett, W. D., Bahcall, J. N., Kirshner, R. P., \& Woosley, S. E. 1989, ARA\&A, 27, 629

Asakura, K., Gando, A., Gando, Y., et al. 2016, ApJ, 818, 1

Audi, G., Wang, M., Wapstra, A. H., et al. 2012, ChPhC, 36, 12

Beacom, J. F., \& Vagins, M. R. 2004, PhRvL, 93, 171101

Braaten, E., \& Segel, D. 1993, PhRvD, 48, 1478

Bruenn, S. W. 1985, ApJS, 58, 771

Burrows, A., \& Lattimer, J. M. 1988, PhR, 163, 51

Buyukcizmeci, N., Botvina, A. S., Mishustin, I. N., et al. 2013, NuPhA, 907, 13

Couch, S. M., Chatzopoulos, E., Arnet, W. D., \& Timmes, F. X. 2015, ApJL, 808,1

Doherty, C. L., Pons, P. G., Siess, L., \& Lattanzio, J. C. 2017, arXiv:1703. $06895 \mathrm{v} 1$

DUNE collaboration 2016, arXiv:1601.05471v1

Fischer, T., Martínez-Pinedo, G., Hempel, M., \& Liebendörfer, M. 2012, PhRvD, 85, 083003

Fuller, G. M., Fowler, W. A., \& Newman, M. J. 1985, ApJ, 293, 1

Furusawa, S., Nagakura, H., Sumiyoshi, K., Kato, C., \& Yamada, S. 2017, PhRvC, 95, 025809
Furusawa, S., Sumiyoshi, K., Yamada, S., \& Suzuki, H. 2013, ApJ, 772, 95 Furusawa, S., Sumiyoshi, K., Yamada, S., \& Suzuki, H. 2017, NuPhA, 957, 188

Guo, G., \& Qian, Y. Z. 2016, PhRvD, 94, 043005

Hirata, K., Kajita, T., Koshiba, M., et al. 1987, PhRvL, 58, 14

Itoh, N., Hayashi, H., Nishikawa, A., \& Kohyama, Y. 1996, ApJS, 102, 411

Janka, H. T. 2012, ARNPS, 62, 407

Janka, H. T., Langanke, K., Marek, A., Martínez-Pinedo, G., \& Müller, B. 2007, PhR, 442, 38

Jones, S., Hirschi, R., Nomoto, K., et al. 2013, ApJ, 772, 150

Juodagalvis, A., Sampaio, J. M., Langanke, K., \& Hix, w. R. 2008, JPhG, 35, 014031

KamLAND Collaboration 2009, JINST, 4, P04017

Kato, C., Azari, M. D., Yamada, S., et al. 2015, ApJ, 808, 168

Kitaura, F. S., Janka, H. T., \& Hillebrandt, E. 2006, A\&A, 450, 345

Kotake, K., Takiwaki, T., Suwa, Y., et al. 2012, AdAst, 428757

Koura 2004, in AIP Proc. 704, TOURS Symp. on Nuclear Physics V, ed. M. Arnould et al. (Melville, NY: AIP), 60

Koura, H., Tachibana, T., Uno, M., \& Yamada, M. 2003, RIKEN Accel Prog. Rep., 36, 9

Koura, H., Uno, M., \& Yamada, M. 2005, PThPh, 113, 305

Kunugise, T., \& Iwamoto, K. 2007, PASJ, 59, 57

Kurganov, A., \& Tadmor, E. 2000, JCoPh, 160, 241

Langanke, K., \& Martínez-Pinedo, G. 2001a, ADNDT, 79, 1

Langanke, K., Martínez-Pinedo, G., \& Sampaio, J. M. 2001b, PhRvC, 64, 055801

Langanke, K., Martínez-Pinedo, G., Sampaio, J. M., et al. 2003, PhRL, 90 , 241102

Liebendöfer, M., Messer, O. E. B., Mezzacappa, A., et al. 2004, ApJS, 150,263

Mezzacappa, A., \& Bruenn, S. W. 1993a, ApJ, 410, 740

Mezzacappa, A., \& Bruenn, S. W. 1993b, ApJ, 405, 669

Misch, G. W., \& Fuller, G. M. 2016, PhRvC, 94, 055808

Müller, B. 2015, MNRAS, 453, 287

Nagakura, H., Ito, H., Kiuchi, K., \& Yamada, S. 2011, ApJ, 731, 80

Nagakura, H., Iwakami, W., Furusawa, S., et al. 2016, arXiv:1605.00666v1

Nagakura, H., Sumiyoshi, K., \& Yamada, S. 2014, ApJS, 214, 16

Nagakura, H., \& Yamada, S. 2008, ApJ, 689, 391

Nomoto, K., \& Hashimoto, M. 1988, Phys. Rep. Lett., 163, 13

Nomoto, K., Sparks, W. M., Fesen, R. A., et al. 1982, Natur, 299, 803

Oda, T., Hino, M., Muto, K., Takahara, M., \& Sato, K. 1994, ADNDT, 56, 231

Odrzywolek, A. 2007, EPJC, 52, 425

Odrzywolek, A. 2009, PhRvC, 80, 045801

Odrzywolek, A., \& Heger, A. 2010, AcPPB, 41, 1611

Odrzywolek, A., Misiaszek, M., \& Kutschera, M. 2004, APh, 21, 303

Particle Data Group 2014, ChPhC, 38, 090001

Patton, K. M., Lunardini, C., \& Farmer, R. J. 2017, arXiv:1511.02820v2

Raduta, A. R., Gulminelli, F., \& Oertel, M. 2016, PhRvC, 93, 025803

Raffelt, G. G. 2012, arXiv:1201.1637

Ravenhall, D. G., Pethick, C. J., \& Wilson, J. R. 1983, PhRvL, 50, 2066

Sato, K., \& Suzuki, H. 1987, PhRvL, 58, 2722

Schinder, P., \& Shapiro, S. 1982, ApJS, 50, 23

Shen, H., Toki, H., Oyamatsu, K., \& Sumiyoshi, K. 2011, ApJS, 197, 2

"SNOwGLoBES," http://www.phy.duke.edu/ schol/snowglobes/

Sullivan, C., O’Connor, E., Zegers, R. T., Grubb, T., \& Austin, S. M. 2016, $\mathrm{ApJ}, 816,44$

Sumiyoshi, Y., \& Yamada, S. 2012, ApJS, 199, 1

Sumiyoshi, Y., Yamada, S., Suzuki, H., et al. 2005, ApJ, 629, 922

Super-Kamiokande collaboration 2014, NIMPA, 737, 253

Tachibana, T. 2000, RIKEN Review 26, 109

Tachibana, T., \& Yamada, M. 1995, in Proc. Inc. Conf. on Exotic Nuclei and Atomic Masses, Arles, ed. M. de Saint Simon \& O. Sorlin (Gif-sur-Yvette: Ed. Frontières), 763

Takahashi, K., Yoshida, T., \& Umeda, H. 2013, ApJ, 771, 28

Takahashi, K., Yoshida, T., Umeda, H., Sumiyoshi, K., \& Yamada, S. 2016, MNRAS, 456, 1320

Tominaga, N., Blinnikov, S. I., \& Nomoto, K. 2013, ApJL, 771, 12

Umeda, H., Yoshida, T., \& Takahashi, K. 2012, PTEP, 01A302

Woosley, S. E., Heger, A., \& Weaver, T. A. 2002, RvMP, 74, 1015

Yoshida, T., \& Tachibana, T. 2000, WJNST, 37, 491

Yoshida, T., Takahashi, K., Umeda, H., \& Ishidoshiro, K. 2016, PhRvD, 93, 123012

Zuber, K. 2015, NPPP, 265, 233 